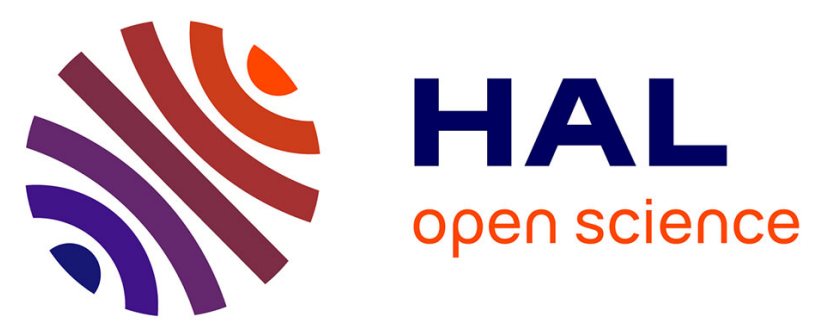

\title{
Determination of the throat size distribution of a porous medium as an inverse optimization problem combining pore network modeling and genetic and hill climbing algorithms
}

\author{
Otman Maalal, Marc Prat, René Peinador, Didier Lasseux
}

\section{To cite this version:}

Otman Maalal, Marc Prat, René Peinador, Didier Lasseux. Determination of the throat size distribution of a porous medium as an inverse optimization problem combining pore network modeling and genetic and hill climbing algorithms. Physical Review E : Statistical, Nonlinear, and Soft Matter Physics, 2021, 10.1103/PhysRevE.103.023303 . hal-03146345

\author{
HAL Id: hal-03146345 \\ https://hal.science/hal-03146345
}

Submitted on 19 Feb 2021

HAL is a multi-disciplinary open access archive for the deposit and dissemination of scientific research documents, whether they are published or not. The documents may come from teaching and research institutions in France or abroad, or from public or private research centers.
L'archive ouverte pluridisciplinaire HAL, est destinée au dépôt et à la diffusion de documents scientifiques de niveau recherche, publiés ou non, émanant des établissements d'enseignement et de recherche français ou étrangers, des laboratoires publics ou privés. 


\title{
Determination of the throat size distribution of a porous medium as an inverse optimization problem combining pore network modeling and genetic and hill climbing algorithms
}

\author{
Otman Maalal $\odot,{ }^{1,2}$ Marc Prat $\odot,{ }^{1, *}$ René Peinador, ${ }^{2}$ and Didier Lasseux $\oplus^{3}$ \\ ${ }^{1}$ Institut de Mécanique des Fluides de Toulouse, Université de Toulouse, Centre National de la Recherche Scientifique, 31400 Toulouse, France \\ ${ }^{2}$ Institut de La Filtration et des Techniques Séparatives, Rue Marcel Pagnol, 47510 Foulayronnes, France \\ ${ }^{3}$ I2M, UMR 5295, Centre National de la Recherche Scientifique, Université Bordeaux, Esplanade des Arts et Métiers, \\ 33405 Talence CEDEX, France
}

(Published 9 February 2021)

\begin{abstract}
The pore size distribution of a porous medium is often estimated from the retention curve or the invading fluid flow rate curve using simple relationships more or less explicitly based on the consideration that the porous medium is made of a bundle of cylindrical parallel tubes. This type of determination is tested using pore network simulations. Starting from two- or three-dimensional networks, the characteristics of which are known a priori, the estimation of the throat size distribution (TSD) is performed using the standard methods in the case of drainage. Results show a significant discrepancy with the input data. The disagreement is more pronounced when the fluid flow rate curve is employed together with the parallel tubes assumption. The physical origins of these shortcomings are identified. A method, based on pore network simulations combined with a genetic algorithm and the hill climbing algorithm, is then designed, which makes simultaneous use of the nonwetting fluid flow rate curve and the retention curve of the medium. Very significant improvement is achieved in the estimation of the TSD using this procedure.
\end{abstract}

DOI: 10.1103/PhysRevE.103.023303

\section{INTRODUCTION}

The pore size distribution (PSD) is a key structure characteristic of a porous medium of major importance to model flow and transport processes. A simple method for determining the PSD is to use the retention curve, i.e., the fluid potential to saturation relationship [1]. As discussed for instance in [2,3], this is often performed assuming more or less explicitly that the pore system can be represented by a bundle of parallel cylindrical capillary tubes of circular cross section with a random distribution of radii. However, except for a few particular systems, for example the Anopore membrane discussed in [4], the microstructure of a porous medium is generally significantly different from a simple bundle of parallel cylindrical tubes. Most porous materials are rather characterized by a system of highly interconnected pores. As a result, the information on the PSD obtained from simple interpretation based on this representation is questionable and must be cautiously assessed [3]. Another issue is that the PSD obtained in this way makes no difference between the throats, i.e., the constrictions in the pore space, and the pore bodies, defined here as the local larger cavities. Making such a distinction between pore throats and pore bodies is a classical feature of pore network models (PNMs) [5]. This is justified by the fact that constrictions are largely controlling the capillary effects since menisci tend to get preferentially pined in constrictions. They are also decisive for transport phenomena since, for example, the pressure drop between two adjacent pore bodies

${ }^{*}$ Corresponding author: mprat@imft.fr can be essentially controlled by the constriction connecting them when this constriction is sufficiently narrow compared to the pore body size [6,7]. This distinguishes the PSD, which corresponds to the pore bodies in the PNM partition of the pore space, from the throat size distribution (TSD), i.e., the distribution of constriction sizes. In this context, the question arises as to whether it is possible to identify both the PSD and TSD, or at least the TSD, from relatively basic information such as the retention curve or the flow rate curve, the latter denoting the flow rate to fluid potential relationship. With the development of imaging techniques, e.g., micro-x-ray tomography, this approach may be itself questioned since the TSD and the PSD can be determined by means of various algorithms applied to segmented images of the microstructure $[8,9]$. However, imaging devices are expensive and the segmentation of the pore space in pore bodies and pore throats is rarely straightforward, requiring the treatment of a huge amount of data. Moreover, obtaining reliable images for materials with pore bodies and throats in the submicronic range is not an easy task. As a result, cheaper and more routine characterization techniques are still quite desirable.

In what follows, the focus is laid on the TSD. The intrinsic permeability and porosity, together with the retention and nonwetting fluid flow rate curves, are supposed to be known, i.e., are available from measurement for a pair of fluids for which the wetting fluid is perfectly wetting. In addition to the assessment of two classical TSD extraction methods, the objective of the paper is to explore whether this information is sufficient to characterize the TSD. Taking into account the nonwetting fluid flow rate is motivated by the fact that the evaluation of a porosimetry technique, referred to as the 
fluid-fluid displacement porosimetry (FFDP) [10,11], is also of interest. In this technique, a perfectly wetting liquid is displaced by a nonwetting one through a series of pressure steps and the nonwetting flow rate through the sample is recorded for each pressure step. The flow rate vs pressure curve is then harnessed to extract the TSD [10]. In the context of FFDP, the situation under study in the present paper can actually be seen as a quasistatic version, referred to as QSFFDP, where QS stands for quasistatic. Contrary to the classical FFDP technique where the viscous pressure drop across the sample in the nonwetting fluid is on the order of the capillary pressure, the viscous pressure drop remains small compared to the capillary pressure. Actually, this corresponds to the situation considered in practice for determining the nonwetting fluid relative permeability. Considering the flow rate curve so as to use the same convention as in the FFDP or the nonwetting fluid relative permeability curve is strictly equivalent in the quasistatic limit under consideration here. The classical FFDP (as opposed to the QSFFDP) will be the object of a future study.

On this basis, the idea is then to rely on pore network simulations. The approach is similar to the one presented in [3]. Starting from a perfectly known microstructure with interconnected pore bodies, referred to as the reference network, an immiscible displacement is performed numerically for conditions similar to the ones employed for the classical retention curve measurement. The TSD is extracted from the retention curve and flow rate data using classical extraction methods based on the bundle of capillary tubes model. The TSD obtained with this procedure is compared to the actual TSD. The decisive feature here is that the TSD is perfectly known a priori for the reference medium. This allows illustrating and assessing the limitations of the classical methods for the TSD determination. Then, the question arises as to how the use of the immiscible displacement data can be improved when the microstructure is clearly not a simple system of parallel tubes. This amounts to considering an ill-posed inverse problem in which the objective is to determine the TSD. Regarding the method used to compute the displacement in the network, main possibilities can be divided into direct simulations like lattice Boltzmann methods [12] or volume of fluid models [13], etc., and PNMs [5]. As will be explained below, the TSD identification method requires computing the immiscible displacement many times. In this respect, PNMs are well adapted as this technique requires much shorter computational time compared to direct simulations.

The present paper is organized as follows. In Sec. II, two classical methods for determining the PSD (more exactly the TSD) from the nonwetting fluid flow rate curve or the retention curve are recalled. The pore network models are presented in Sec. III. The algorithms used to simulate the displacement and compute the flow rate and retention curves are presented in Sec. IV. The assessment of the TSD determination from the classical methods is presented in Sec. V. In Sec. VI, a method to determine the TSD from both the nonwetting fluid normalized flow rate and retention curves using PNM simulations combined with an optimization method is presented. A short discussion is presented in Sec. VII. Conclusions are drawn in Sec. VIII.

\section{FLOW RATE POROSIMETRY AND RETENTION CURVE POROSIMETRY}

Suppose the retention curve, $P_{c}(S)$, flow rate curve, $Q_{\mathrm{NW}}$ $\left(P_{c}\right)$, and porosity, $\varepsilon$, are known, $P_{c}(S)$ being the capillary pressure, $Q_{\mathrm{NW}}$ the nonwetting fluid flow rate for the quasistatic fluid distribution corresponding to $P_{c}(S)$, and $S$ the wetting fluid saturation. Considering the generalized Darcy's law implies

$$
Q_{\mathrm{NW}}=A \frac{k k_{r}\left(P_{c}\right)}{\mu} \frac{\Delta P}{L}
$$

where $\mu$ is the nonwetting fluid viscosity, $A$ is the porous medium cross-sectional surface area, $L$ is the sample length, $k$ is the porous medium intrinsic permeability, and $k_{r}$ is the nonwetting fluid relative permeability. In addition, $\Delta P$ is the pressure difference between the inlet and outlet in the nonwetting fluid. In the quasistatic limit under consideration here, $\Delta P$ is small compared to the capillary pressure. Throughout the paper, a constant $\Delta P$ is considered independently of the capillary pressure.

To extract the TSD from the retention curve, a pore size, $r$, is associated to a value of $P_{c}$ using the Young-Laplace equation

$$
P_{c}=\frac{2 \gamma \cos \theta}{r}
$$

where the contact angle is $\theta=0$ in our case (perfectly wetting displaced fluid) and $\gamma$ is the interfacial tension. For simplicity, trapping phenomena [14] are assumed negligible so that $S$ varies in the interval $[0,1]$. The wetting fluid saturation, $S$, corresponding to $r$ is then simply defined as [15]

$$
S=\frac{\int_{0}^{r} V_{p}(r) f(r) d r}{\int_{0}^{+\infty} V_{p}(r) f(r) d r}
$$

where $V_{p}(r)$ is the volume of a pore of size $r$ and $f(r)$ is the pore size distribution, i.e., the pore size probability density function (p.d.f.). Deriving Eq. (3) with respect to $r$ yields

$$
\frac{d S}{d r}=\frac{V_{p}(r) f(r)}{\int_{0}^{+\infty} V_{p}(r) f(r) d r} .
$$

Since, by definition, $\int_{0}^{+\infty} V_{p}(r) f(r) d r=\varepsilon V_{\mathrm{PM}}$, where $V_{\mathrm{PM}}$ is the porous medium volume, this leads to

$$
f(r)=\frac{\varepsilon V_{\mathrm{PM}}}{V_{p}(r)} \frac{d S}{d r} .
$$

In discrete form, Eq. (5) can be expressed as

$$
f_{k}\left(r_{A B}\right)=\frac{\varepsilon V_{\mathrm{PM}}}{V_{p}\left(r_{A B}\right)}\left(\frac{S\left(P_{c B}\right)-S\left(P_{c A}\right)}{r_{B}-r_{A}}\right)
$$

where $P_{c A}$ and $P_{c B}$ are two close values of $P_{c}$ and $r_{A B}=$ $\left(r_{A}+r_{B}\right) / 2, r_{A}$ and $r_{B}$ being determined from Eq. (2). Within the framework of the model of a bundle of parallel cylindrical tubes, the volume of a pore is expressed as $V_{p}(r)=\pi r^{2} L$ where $L$ is the tube length assuming straight tubes. Under these circumstances, Eq. (6) is expressed as

$$
f_{k}\left(r_{A B}\right)=\frac{\varepsilon V_{\mathrm{PM}}}{\pi L} r_{A B}^{-2}\left(\frac{S\left(P_{c B}\right)-S\left(P_{c A}\right)}{r_{B}-r_{A}}\right) .
$$


The value of $f_{k}$ is then normalized according to

$$
d_{k}\left(r_{A B}\right)=\frac{f_{k}}{\sum_{i=1}^{n} f_{i}} .
$$

Here, $n$ is the number of classes considered to compute the discrete p.d.f. Hence, $d_{k}\left(r_{A B}\right)$ represents the fraction of pores (number of pores divided by total number of pores) the sizes of which are in class $k$.

The method for determining the TSD from the flow rate $Q_{\mathrm{NW}}\left(P_{c}\right)$ is similar and also explicitly relies on the model of a bundle of parallel cylindrical tubes. It is also similar to the one used in the FFDP technique [10]. In the bundle of tubes model, the flow rate is computed applying Poiseuille's law in each tube containing the nonwetting fluid:

$$
Q_{\mathrm{NW}}=\frac{\Delta P \pi}{8 \mu L} \int_{r_{\min }}^{+\infty} r^{4} f_{Q}(r) d r
$$

where the tube length is again $L$ assuming straight tubes and

$$
r_{\min }=\frac{2 \gamma \cos \theta}{P_{c}}
$$

$f_{Q}$ being again the TSD. In principle, $f_{Q}=f$. However, a different notation is used since it is not obvious that both procedures lead to the same TSD when the porous medium microstructure is different from a bundle of parallel tubes.

Combining Eqs. (1) and (10), assuming $\Delta P=$ const $\ll P_{c}$ independently of the capillary pressure, and deriving the ensuing equation with respect to $r$ lead to

$$
\frac{d Q_{\mathrm{NW}}}{d r}=-\frac{\Delta P \pi}{8 \mu L} r^{4} f_{Q}(r)
$$

and thus

$$
f_{Q}(r)=-\frac{8 \mu L}{\Delta P \pi} r^{-4} \frac{d Q_{\mathrm{NW}}}{d r}
$$

Equation (12) can be expressed in discrete form as [10]

$$
f_{Q k}\left(r_{A B}\right)=-\frac{8 \mu L}{\Delta P \pi} r_{A B}^{-4}\left(\frac{Q_{\mathrm{NW}_{B}}-Q_{\mathrm{NW}_{A}}}{r_{B}-r_{A}}\right) .
$$

The value of $f_{Q k}$ is then normalized as

$$
d_{Q k}\left(r_{A B}\right)=\frac{f_{Q k}}{\sum_{i=1}^{n} f_{Q i}}
$$

In what follows, water is considered as the wetting fluid and air as the displacing fluid. Thus $\gamma=0.072 \mathrm{~N} / \mathrm{m}$ and $\mu=$ $\mu_{\mathrm{nw}}=10^{-5} \mathrm{~Pa} \mathrm{~s}$.

\section{INTERCONNECTED MODEL POROUS MEDIUM}

The QS immiscible displacement is simulated considering simple model porous media referred to as PNMs. In a PNM, the pore space is represented as a network of pore bodies connected by narrower channels. The pore bodies correspond to locally larger volumes whereas the channels correspond to the throats of the pore space, i.e., the narrower passages, also referred to as the constrictions, between pore bodies. Although it is possible to construct the network from digital images of real microstructures (see, e.g., [16]), simpler networks are sufficient for testing the QSFFDP. In what follows,

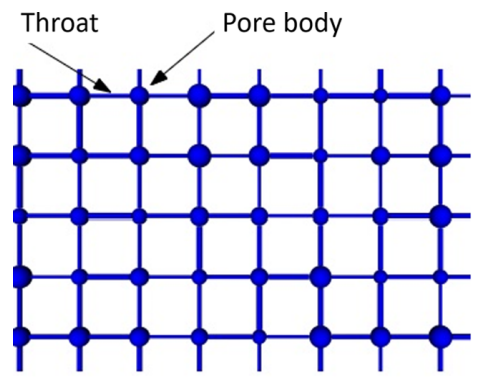

(a)

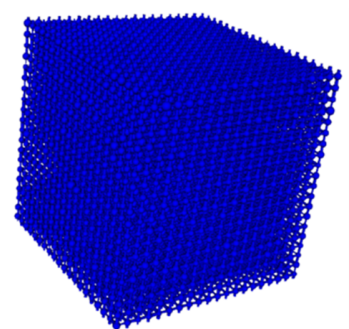

(b)
FIG. 1. Square (2D) (a) and cubic (3D) (b) pore networks.

square [two-dimensional (2D)] and cubic [three-dimensional (3D)] networks are considered. As illustrated in Fig. 1, pore bodies are spheres of radius $r_{p}$ located at the nodes of a regular grid. The distance between two adjacent nodes is the lattice spacing, $a$, with $a=350 \mu \mathrm{m}$ in the simulations presented below. The size of the network is the number of nodes (pore bodies) in each direction [for example, Fig. 1(a) shows a $8 \times 5$ square network]. Throats are cylindrical channels of radius $r_{t}$. The throat size is randomly distributed according to a given p.d.f. Similarly, the pore body size can be distributed according to a given p.d.f. with the constraint that the pore body size is equal to or greater than the largest throat to which it is connected. In what follows, several types of p.d.f. will be considered, namely, uniform, Gaussian, log normal, and bimodal. Also, it can be noted that the network coordination number, i.e., the number of throats connected to a pore body, is 4 for the $2 \mathrm{D}$ square network and 6 for the $3 \mathrm{D}$ cubic network.

\section{FLUID-FLUID IMMISCIBLE QUASISTATIC DISPLACEMENT SIMULATION}

Modeling of immiscible two-phase flow displacements on a network has been the subject of many works and the reader is referred to $[5,17,18]$ for reviews. Here, the interest is on the displacement of a wetting fluid by a nonwetting one in the network. This process is known as drainage. In the quasistatic limit considered for the present paper, it was shown that drainage can be simulated using a simple algorithm referred to as the invasion percolation (IP) algorithm [19-21]. This algorithm can be summarized as follows. A pressure difference, $P_{\mathrm{ck}}$, between the two phases is applied by imposing a pressure increment in the nonwetting phase in contact with one face of the network, identified as the inlet face. The opposite face is the outlet, whereas all the other faces of the network are kept impervious. At each pressure increment, all the throats containing a meniscus for which the capillary pressure threshold, $P_{c \text { th }}$, as expressed by Eq. (2), is such that $P_{\mathrm{ck}} \geqslant P_{c \mathrm{th}}$ are identified. The throat for which $\left(P_{\mathrm{ck}}-P_{c \mathrm{th}}\right) / P_{\mathrm{ck}}$ is maximum, hence corresponding to the interfacial throat of maximum diameter among the interfacial throats such that $\frac{\left(P_{\mathrm{ck}}-P_{c \mathrm{th}}\right)}{P_{\mathrm{ck}}}>0$, is invaded together with the adjacent pore body if the latter was not already invaded in a previous step. If this pore body was occupied by the wetting fluid, new menisci are then positioned at the entrance of the throats occupied by the wetting fluid and connected to the newly invaded pore body. In other words, the list of interfacial throats is updated 
so as to remove the invaded throat and add the throats connected to the newly invaded pore body and occupied by the wetting fluid. This procedure is repeated until there are no unstable menisci remaining in the network, i.e., until there exists no throat for which $\frac{\left(P_{\mathrm{ck}}-P_{\mathrm{cth}}\right)}{P_{\mathrm{ck}}}>0$. The resulting wetting - nonwetting fluid distribution corresponds to the equilibrium distribution at the specified value of $P_{\mathrm{ck}}$. For a sufficiently low pressure difference between the two fluids, the wetting phase displacement is not sufficient for the invading phase to reach the network outlet. The first equilibrium for which the invading fluid reaches the outlet corresponds to the breakthrough (BT) and is characterized by $P_{\mathrm{ck}}=P_{c \mathrm{BT}}$. While incrementing the displacing fluid pressure, the fluid distribution at BT is the first for which the nonwetting fluid can flow through the network, which means that $Q_{\mathrm{NW}}=0$ for $P_{\mathrm{ck}}<P_{c \mathrm{BT}}$ while $Q_{\mathrm{NW}}>0$ for $P_{\mathrm{ck}} \geqslant P_{c \mathrm{BT}}$. Note that the invasion rules described above correspond to a quasistatic drainage process without trapping. This means that an element, pore body, or throat, occupied by the wetting fluid, is assumed to be always connected to the outlet face, possibly through liquid films [22] present in the pore body and throat surface roughness and/or in a subnetwork of small crevices at the throat and pore body walls. Trapping rules could be implemented (see for instance [14]), but, for simplicity, they are not considered in what follows. It can be argued that neglecting trapping is not consistent with the considered cylindrical throat shape since this shape favors trapping phenomena as opposed to throats of a polygonal cross section which favor corner flows [22] and thus the wetting fluid hydraulic connectivity throughout the network. However, what is really important within the scope of the present paper is to consider the same flow physics in the reference network and the networks in the optimization procedure (see Sec. VI). The idea is to keep the simulations as simple as possible in the present effort. For this reason, trapping phenomena have been neglected without explicitly considering corner flows and thus throats of a polygonal cross section.

Each pressure increment provides a point in the graph of the retention curve, the fluid potential being the capillary pressure (i.e., the pressure difference between the nonwetting and wetting fluids) and the saturation being computed from the equilibrium fluid distribution. Next, the computation of the nonwetting fluid flow rate, $Q_{\mathrm{NW}}$, after breakthrough is performed as follows [5]. At each saturation equilibrium, a pressure difference, $\Delta P$, much smaller than the capillary pressure characterizing this equilibrium, is applied in the nonwetting fluid between the network inlet and outlet. For the resulting flow, the mass conservation equation is expressed at each invaded pore body $i$ :

$$
Q_{\mathrm{NW} i, j}=0
$$

where $Q_{\mathrm{NW} i, j}$ is the nonwetting fluid volume flow rate between pore body $i$ and adjacent pore body $j$. If the throat between pore bodies $i$ and $j$ is occupied by the wetting fluid then $Q_{\mathrm{NW}} i, j=0$. If the throat is occupied by the nonwetting fluid, then

$$
Q_{\mathrm{NW} i, j}=\frac{\pi r_{t i, j}^{4}}{8 \mu_{\mathrm{NW}} l_{i, j}} \Delta P_{i, j}
$$

where $r_{t i, j}$ and $l_{i, j}$ are the radius and length of the throat linking the two pore bodies, respectively, while $\Delta P_{i, j}$ is the pressure drop in the nonwetting phase between the two neighboring pore bodies. Taking into account the pressure boundary conditions at the inlet $\left(P_{\text {inlet }}=\Delta P+P_{\text {outlet }}\right)$ and outlet $\left(P_{\text {outlet }}=C t e\right)$ and the zero flux condition imposed on the lateral faces of the network, Eqs. (15) and (16) lead to a linear system for the pressure field in the nonwetting fluid. This system is numerically solved using the conjugate gradient method. Once the pressure field is obtained, $Q_{\mathrm{NW}}$ can be computed either at the outlet or inlet using Poiseuille's law applied to all nonwetting fluid throats connected to the outlet or inlet, respectively.

In summary, the fluid-fluid distribution is computed, in a first step, neglecting the viscous pressure drop in each fluid phase on the grounds that capillary effects are dominant. For this given fluid-fluid distribution, the flow is computed in a second step as indicated above. This is carried out by sampling the capillary pressure, $P_{c}$, with successive increasing values, $P_{\text {ck }}$, the former step allowing the determination of the retention curve and the latter allowing the determination of the flow rate curve. Examples of fluid distribution in a 2D network are reported in Fig. 2.

Figure 3 shows the retention curve and the nonwetting fluid normalized flow rate $Q_{n}=\frac{Q_{\mathrm{NW}}}{Q_{\max }}$ obtained with the above algorithm for a $50 \times 50$ square network with Gaussian distributions for both the TSD and PSD having mean radii of 30 and $90 \mu \mathrm{m}$ and standard deviations of 5 and $10 \mu \mathrm{m}$, respectively. Here, $Q_{\max }$ is the flow rate that would be obtained when the network is fully invaded and the same $\Delta P$ as the one used to compute $Q_{\mathrm{NW}}$ is applied, which means that $Q_{\max }=A \frac{k}{\mu} \frac{\Delta P}{L}$, and thus, from Eq. (1), that $Q_{n}=k_{r}$.

\section{ASSESSMENT OF TSD EXTRACTION FROM THE PARALLEL TUBES MODELS}

As illustrated in Fig. 3, the PNM allows us to simulate the flow rate and retention (or capillary) curves with the decisive advantage that the TSD (and the PSD) of the medium is perfectly known a priori; it is referred to as the reference TSD in the following. Then, starting from the computed curves, the standard determination procedures can be used, i.e., Eqs. (6) and (13), in order to extract the TSD (or PSD) and compare the result with the reference TSD (or PSD). Since the throats control the displacement process, it is reasonable to expect that only the TSD can be determined. The TSD obtained from the parallel tubes model using either Eq. (6) or Eq. (13) is referred to as the simple model TSD, i.e., the SMTSD. A $2 \mathrm{D}$ square $50 \times 50$ network and a $3 \mathrm{D}$ cubic $20 \times 20 \times 20$ network were considered for the comparison.

For each network, the $Q_{n}\left(P_{c}\right)$ and $P_{c}(S)$ curves were computed and the SMTSD extracted using Eqs. (6) and (13). In order to get smoother results, the extracted data, for a given type of network, were averaged over 100 realizations of the network. This corresponds to 100 realizations of each network type for which throat and pore body sizes are randomly assigned according to the given p.d.f. This means that the fluid-fluid displacement was computed 100 times for each case under consideration. Then the SMTSD was extracted from each flow rate curve and each retention curve, yielding 


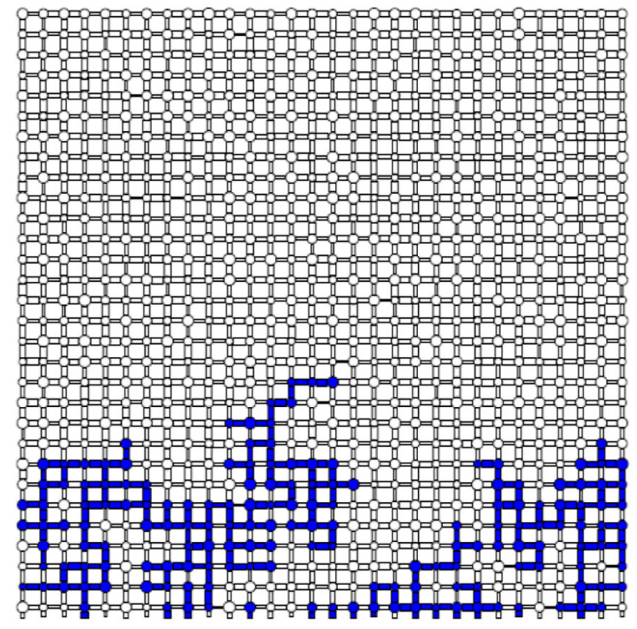

(a)

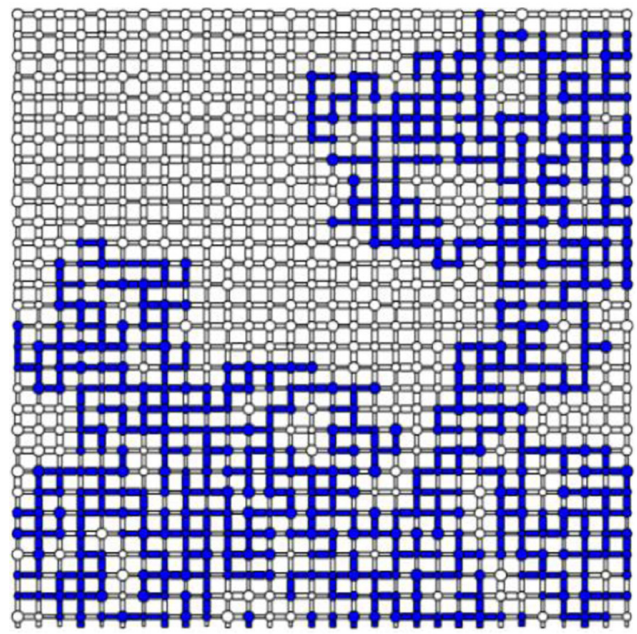

(c)

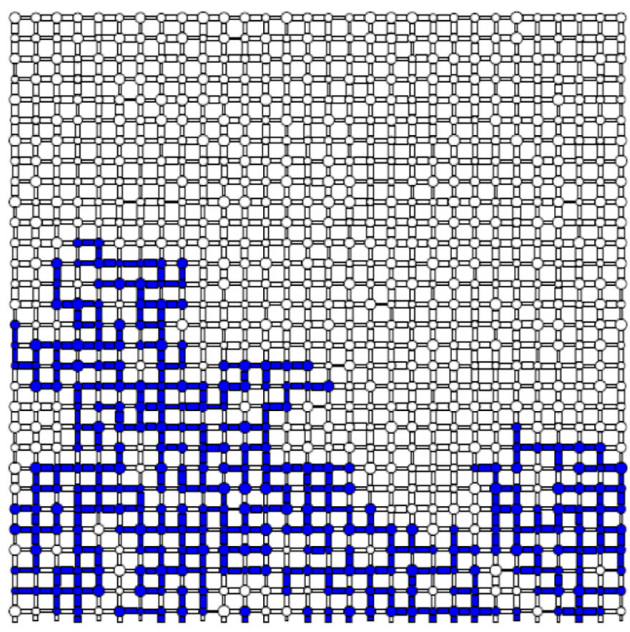

(b)

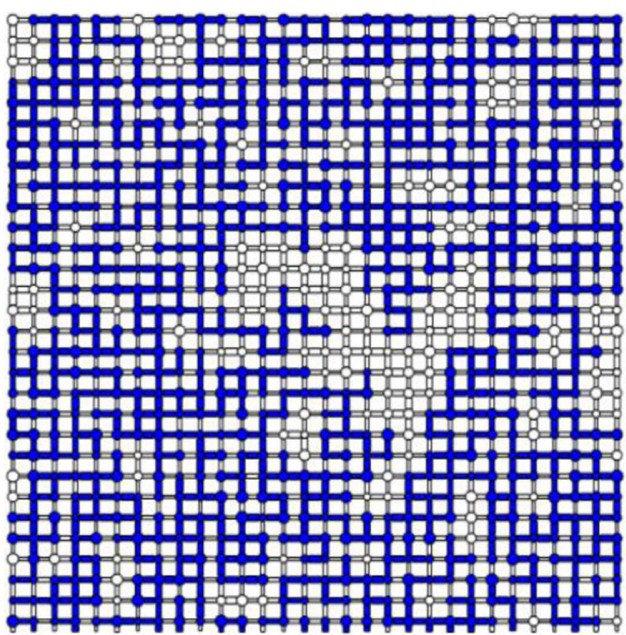

(d)

FIG. 2. Examples of fluid-fluid distribution computed in a $30 \times 302 \mathrm{D}$ network with a uniform TSD characterized by $r_{\min }=20 \mu \mathrm{m}$ and $r_{\max }=60 \mu \mathrm{m}$. Invading fluid in blue enters the network at the bottom face: (a) $P_{c}=2900 \mathrm{~Pa}, S=0.82$, (b) $P_{c}=3250 \mathrm{~Pa}, S=0.71$, (c) $P_{c}=3650 \mathrm{~Pa}, S=0.55$, (d) $P_{c}=4250 \mathrm{~Pa}, S=0.12$.

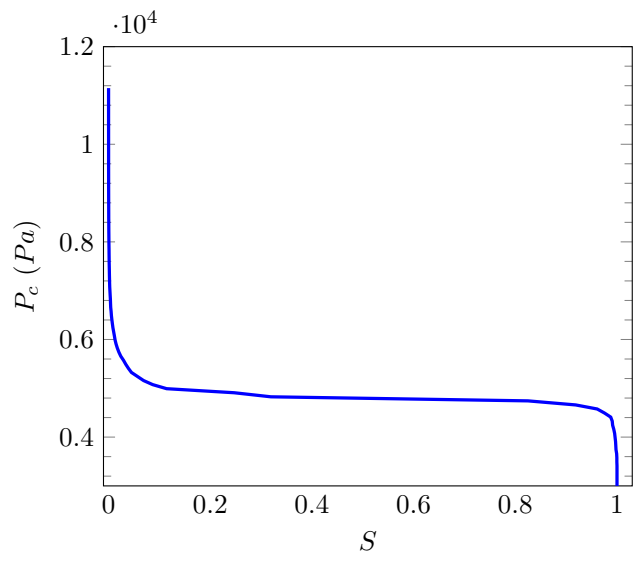

(a)

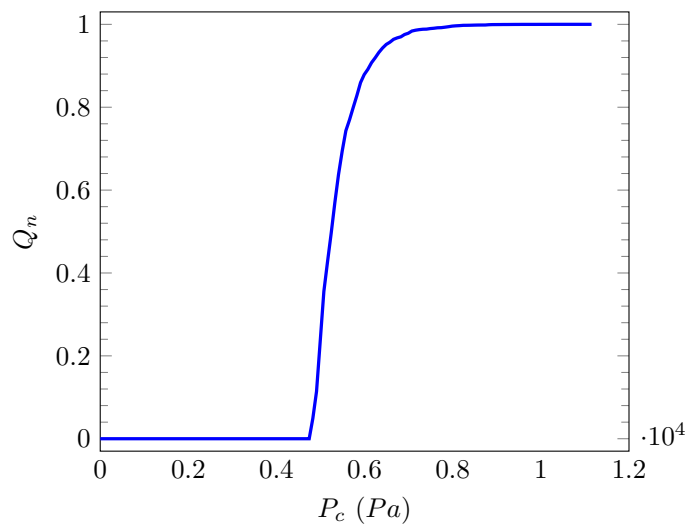

(b)

FIG. 3. Example of computed retention curve (i.e., capillary pressure vs the wetting fluid saturation) (a) and nonwetting fluid normalized flow rate curve (b) for a $50 \times 50$ square network with Gaussian PSD (mean radius, $90 \mu \mathrm{m}$; standard deviation, $10 \mu \mathrm{m}$ ) and TSD (mean radius, $30 \mu \mathrm{m}$; standard deviation, $5 \mu \mathrm{m}$ ). 


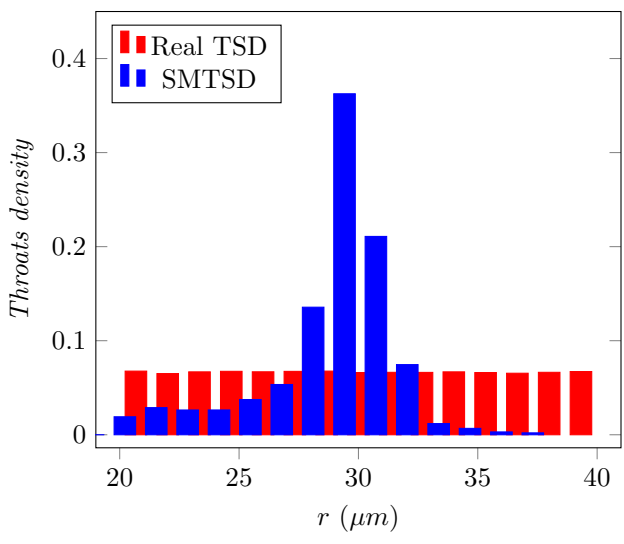

(a)

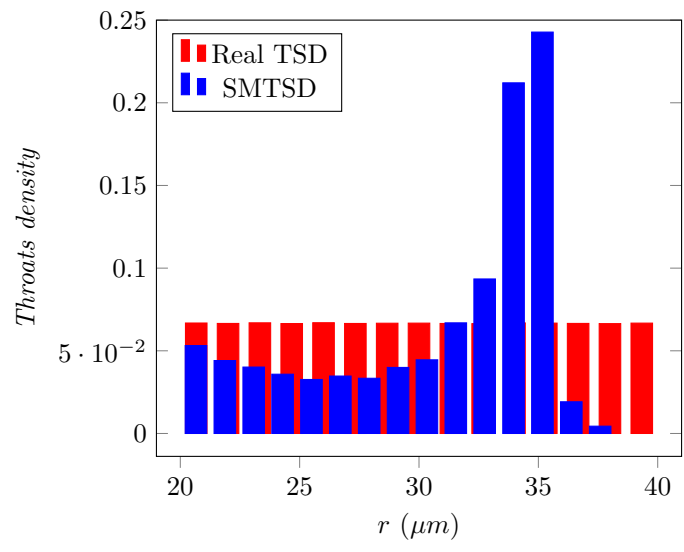

(c)

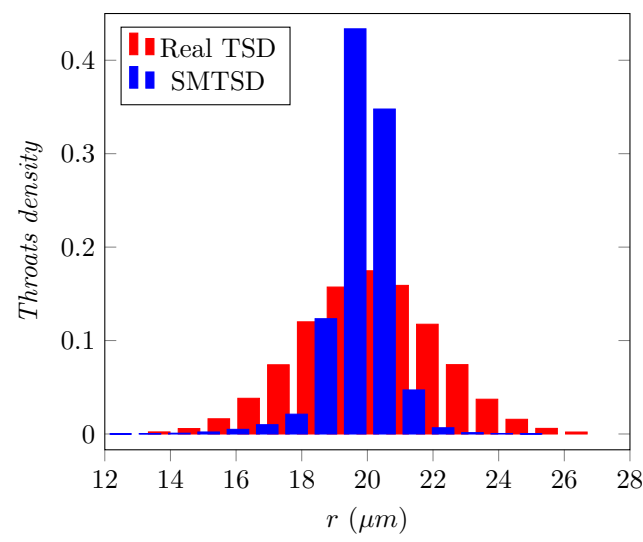

(b)

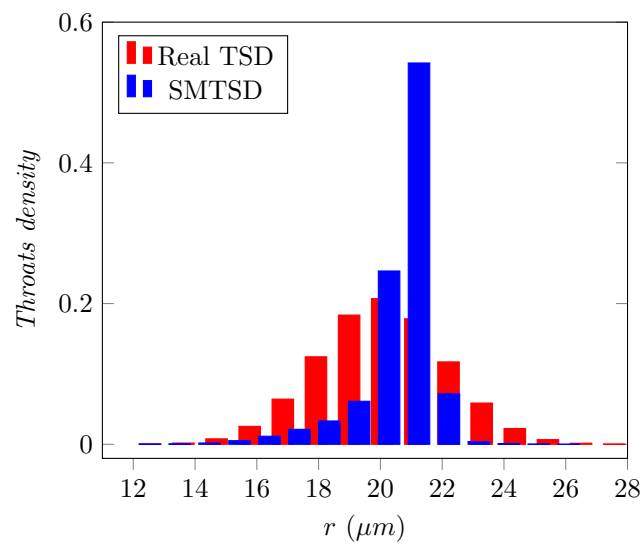

(d)

FIG. 4. Comparison of the TSD obtained from the simple model (SMTSD) using the retention curve $P_{c}(S)$, i.e., Eq. (6), and the reference (real) TSD for various cases: (a) 2D $50 \times 50$ network, uniform p.d.f., $r_{\max }=40 \mu \mathrm{m}, r_{\min }=20 \mu \mathrm{m}$, (b) $2 \mathrm{D} 50 \times 50$ network, Gaussian p.d.f., $r_{\text {moy }}=20 \mu \mathrm{m}, \sigma=2 \mu \mathrm{m}$, (c) $3 \mathrm{D} 20 \times 20 \times 20$ network, uniform p.d.f., $r_{\max }=40 \mu \mathrm{m}, r_{\min }=20 \mu \mathrm{m}$, (d) $3 \mathrm{D} 20 \times 20 \times 20$ network, Gaussian p.d.f., $r_{\text {moy }}=20 \mu \mathrm{m}, \sigma=2 \mu \mathrm{m}$.

$2 \times 100$ TSD. Then, for either the flow rate curves or the retention curves, the average TSD is computed from the 100 TSD. This average is presented and discussed below. A "case" is defined by the dimensionality of the network, i.e., square or cubic; the size of the network; the selected p.d.f., i.e., uniform or Gaussian; and the parameters of the p.d.f., namely, the minimum and maximum radii, $r_{\min }$ and $r_{\max }$, respectively, for the uniform distribution or the mean size and standard deviation, $r_{\text {moy }}$ and $\sigma$, respectively, for the pore bodies and the throats in the case of a Gaussian p.d.f.

The comparison between the reference TSD and the SMTSD for various cases when the retention curve information [Eq. (6)] is used is presented in Fig. 4 and Table I. As can be seen, the SMTSD does not match the reference TSD, in particular for the uniform distribution for which the uniform nature is not retrieved. For the 3D networks, it can be seen that the SM procedure introduces skewness towards large throat size. In brief, the simple model (SM) does not contain the necessary information to predict the reference TSD.

As illustrated in Fig. 5 and Table I, the SMTSD is somewhat less well estimated from the flow rate curve compared to the case where the capillary curve information is used.
In particular, it can be noticed that the larger throats are not identified.

A major shortcoming is that some throat sizes are not retrieved at all. In the case of the $2 \mathrm{D}$ network with a uniform distribution for instance, the throat sizes in the upper half of the distribution are not retrieved, in particular while employing the flow rate curve. Thus, only half of the throat sizes is actually identified. The same problem can be noticed with the $3 \mathrm{D}$ network, but to a lesser extent since the range of nonidentified throat sizes is narrower (but here again the larger throats are not identified). Insights on this result can be gained from percolation theory [23]. In the percolation theory, a certain fraction, $p$, of throats are "active." This fraction of active throats (or "bonds" in the language of percolation theory) is distributed randomly. Suppose $p$ is progressively increased starting from $p=0$. For a particular value, $p_{c}$, of this fraction, a percolating path (a path of interconnected active throats connecting the inlet to the outlet) forms for the first time in the network. The quantity $p_{c}$ is the percolation threshold of the network the value of which depends on the network [23]. For a square network in bond percolation, $p_{c}=0.5$, whereas $p_{c} \approx 0.25$ for a cubic network. 
TABLE I. Comparison between the reference TSD and the SMTSD (parallel tubes) obtained from the Young-Laplace equation and the capillary pressure (or retention) curve, Eq. (6), or from the normalized flow rate curve, Eq. (13), for various cases.

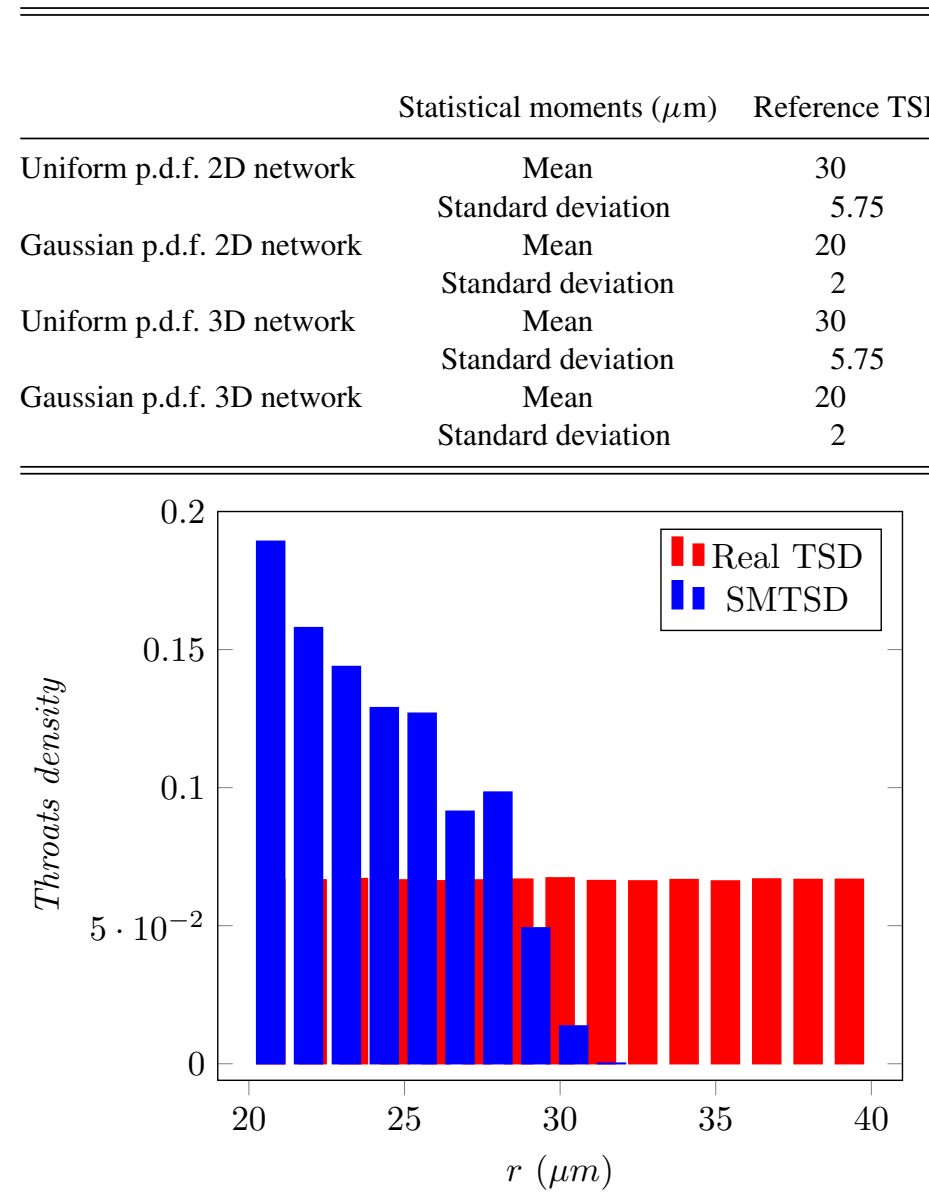

(a)

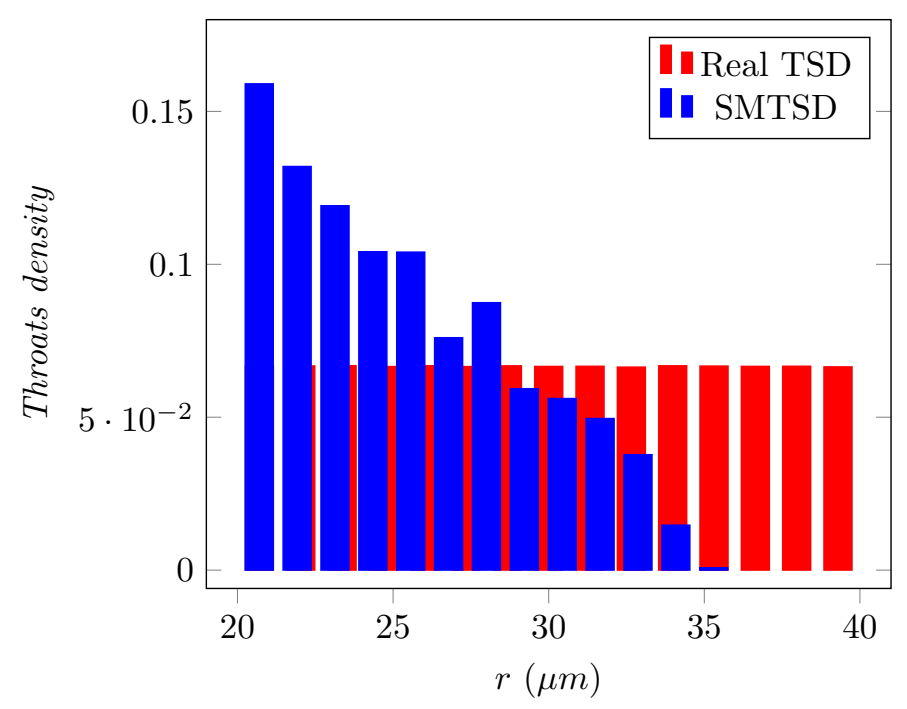

(c)
Parallel tubes model and $P_{c}(S) \quad$ Parallel tubes model and $Q_{n}(S)$

SMTSD Relative error (\%) $\quad$ SMTSD Relative error (\%)

$\begin{array}{rccc}28.84 & 3.88 & 24.14 & 19.53 \\ 2.78 & 48.3 & 2.72 & 52.64 \\ 19.84 & 0.79 & 18.08 & 9.58 \\ 0.92 & 54.04 & 1.4 & 30.1 \\ 30.94 & 3.14 & 25.38 & 15.4 \\ 4.79 & 16.77 & 3.77 & 34.44 \\ 20.69 & 3.45 & 18.6 & 7 \\ 1.19 & 40.5 & 1.59 & 20.3\end{array}$

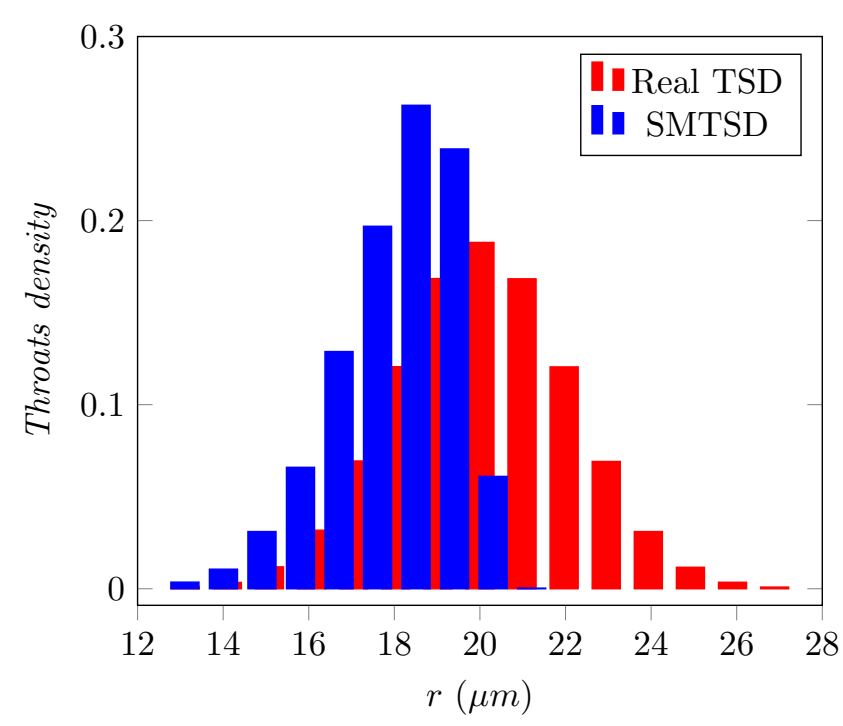

(b)

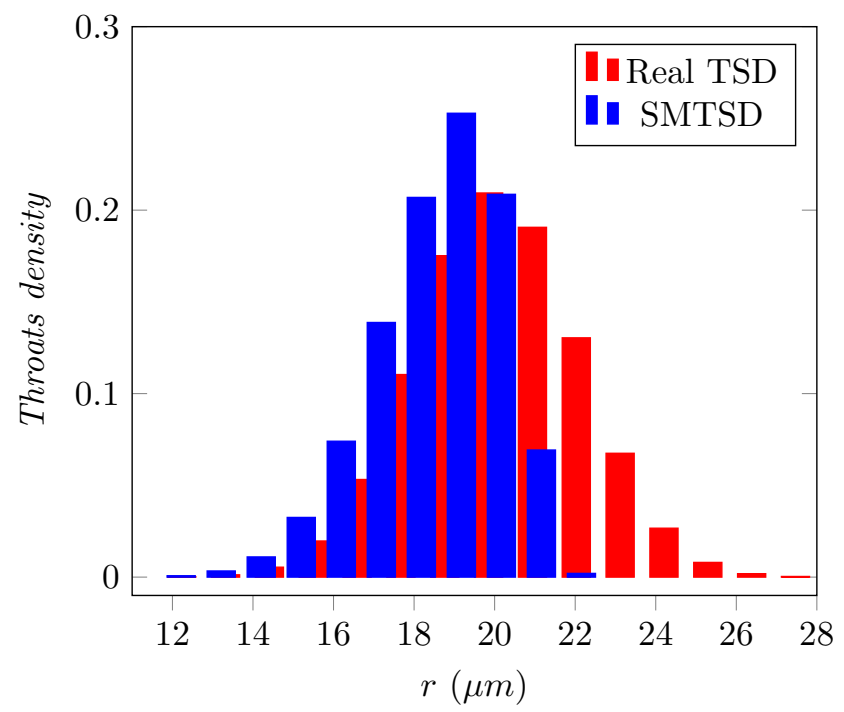

(d)

FIG. 5. Comparison of the TSD obtained from the parallel tubes model (SMTSD) and the reference (real) TSD for various cases using the flow rate information, Eq. (13). $r_{c}$ is the critical radius corresponding to the network percolation threshold: (a) $2 \mathrm{D} 50 \times 50$ network, uniform p.d.f., $r_{\max }=40 \mu \mathrm{m}, r_{\min }=20 \mu \mathrm{m}, r_{c}=30 \mu \mathrm{m}$, (b) $2 \mathrm{D} 50 \times 50$ network, Gaussian p.d.f., $r_{\text {moy }}=20 \mu \mathrm{m}, \sigma=2 \mu \mathrm{m}, r_{c}=20 \mu \mathrm{m}$, (c) $3 \mathrm{D} 20 \times 20 \times 20$ network, uniform p.d.f., $r_{\max }=40 \mu \mathrm{m}, r_{\min }=20 \mu \mathrm{m}, r_{c}=35 \mu \mathrm{m}$, (d) $3 \mathrm{D} 20 \times 20 \times 20$ network, Gaussian p.d.f., $r_{\text {moy }}=$ $20 \mu \mathrm{m}, \sigma=2 \mu \mathrm{m}, r_{c}=21.4 \mu \mathrm{m}$. 
In the present context, an active throat is a throat that can be invaded, i.e., a throat such that $P_{\mathrm{ck}} \geqslant P_{c \text { th }}$, and the breakthrough corresponds to the percolation threshold since there is no nonwetting fluid path flowing through the network before breakthrough. The percolation threshold can thus be related to the TSD:

$$
p_{c}=\int_{r_{c}}^{\infty} f(r) d r=1-\int_{0}^{r_{c}} f(r) d r=1-F\left(r_{c}\right)
$$

where $r_{c}$ is the percolation radius, i.e., the radius given by $P_{c \text { th }}=P_{c \mathrm{BT}}, f$ being the reference TSD p.d.f., and $F$ the corresponding cumulative distribution function [24].

Thus, the threshold or percolation radius, $r_{c}$, represents the critical radius above which the flow rate is nonzero. This means that, when the bundle of parallel tubes model is considered, all the throats of the network having radii greater than the threshold radius, which are hence invaded by the nonwetting fluid prior to the BT, cannot be determined by the parallel tubes model.

From Eq. (17), $r_{c}$ is given by

$$
r_{c}=r_{\max }\left(1-p_{c}\right)+p_{c} r_{\min }
$$

for a uniform p.d.f., where $r_{\max }$ and $r_{\min }$ are the maximum and the minimum radii of the (reference) TSD, respectively. For a Gaussian TSD, the threshold radius is given by

$$
r_{c}=r_{\text {moy }}+\sqrt{2} \sigma \operatorname{erf}^{-1}\left(1-2 p_{c}\right)
$$

where $\operatorname{erf}^{-1}$ is the inverse error function.

For a square lattice $\left(p_{c}=0.5\right)$, the above relationships give $r_{c}=\frac{r_{\max }+r_{\min }}{2}$ and $r_{c}=r_{\text {moy }}$ for the uniform and Gaussian p.d.f., respectively. For a cubic lattice ( $\left.p_{c} \approx 0.25\right)$, one obtains $r_{c}=\frac{3}{4} r_{\max }+\frac{1}{4} r_{\min }$ and $r_{c}=r_{\text {moy }}+\frac{\sqrt{2}}{2} \sigma$ for the uniform and Gaussian p.d.f., respectively. The values are reported in Fig. 5. The analytical threshold radii are perfectly consistent with the results shown in the TSD comparisons in Fig. 5. In summary, the parallel tubes model is not the appropriate representation able to identify the throat sizes greater than the critical radius corresponding to the network percolation threshold. The greater the percolation threshold, the larger the range of missing throats in the TSD determination with the parallel tubes model.

\section{BEYOND THE TSD EXTRACTION FROM THE PARALLEL TUBES MODEL}

The observation above calls upon more sophisticated procedures to extract the TSD of a porous material from data available from a primary drainage process. As shown in the previous section, the standard and commonly used models to extract the TSD do not allow determining the reference TSD from the $Q_{n}\left(P_{c}\right)$ or $P_{c}(S)$ data. This unsatisfactory situation is explained by two reasons. First, for a given pressure step, all the newly invaded throats, regardless of their sizes, are assigned to a single throat size value corresponding to the imposed pressure difference related to the Young-Laplace equation (2). Second, when the determination of the TSD is based on the flow rate curve, all the radius values larger than the percolation radius cannot be determined since the flow rate is zero until breakthrough is reached, hence providing no information on all the throats of radius larger than the critical value. Thus, the question arises as to whether a better use of the $Q_{n}\left(P_{c}\right)$ and $P_{c}(S)$ curves can be performed in order to predict the reference TSD. An attempt in this direction is presented in the following.

\section{A. TSD determination as an inverse problem}

In essence, the problem to be solved is an inverse problem relying on the following question: knowing the $Q_{n}\left(P_{c}\right)$ and $P_{c}(S)$ curves, can the TSD of the corresponding medium be reliably predicted? In this procedure, the direct problem is of course to predict the $Q_{n}\left(P_{c}\right)$ and $P_{c}(S)$ curves of the porous medium under consideration. In addition, the inverse problem is solved using again the PNM and the direct problem is solved as in the previous section using the IP algorithm in the absence of trapping, considering the pressure and no flux boundary conditions already mentioned in Sec. IV. Actually, a somewhat simplified version of this inverse problem is tackled, assuming that the pore network structure is known. This means that the minimization procedure to fit $Q_{n}\left(P_{c}\right)$ and $P_{c}(S)$ is carried out for a given type of network, i.e., square or cubic for instance. In other words, for a given type of network (a $50 \times 502 \mathrm{D}$ square network for instance), the objective is to correctly identify the TSD. The assumption that the pore network structure is known a priori is further discussed in Sec. VII below. To build and test the procedure, a particular realization of the network, with specified p.d.f. parameters, is used as the reference network the TSD of which is hence known. It shall be viewed as the porous medium to be characterized for which the experimental measurement of $Q_{n}\left(P_{c}\right)$ and $P_{c}(S)$ would have been performed in practice and the TSD of which is to be determined.

The inverse problem is solved using an optimization method based on a searching algorithm which is a hybrid version of the genetic and hill climbing algorithms [25]. The genetic algorithm [26,27] is a searching algorithm based on the biological evolution laws including reproduction, mutation, and natural selection. A population of individuals is first defined. In our case, the individuals are realizations of the pore network. Every individual has genes. A gene in a pore network is either a throat or a pore body characterized by its size. The algorithm is relying on a fitness function the value of which is estimated from a direct solution for each individual. The fitness function, denoted fit $\left(\mathbf{r}_{t}\right)$ in its generic form, is an objective function comparing the curve obtained by direct simulation for the individual to the reference one, $\mathbf{r}_{t}$ being a vector the components of which are the individual throat radii. The genetic algorithm aims at minimizing this fitness function [26].

The overall algorithm can be summarized by the following steps.

The first step consists in generating a series of $N_{0}$ parent realizations ( $N_{0}=16$ in this paper) to which evolutionary laws and natural selection are applied. In the most general case, both the throat and pore body sizes vary from one element (pore body or throat) to the other in the network. Referring for instance to the computation of the normalized flow rate, it is clear from Eq. (16) that the local volume flow rate through a throat depends not only on the throat radius, but also on the adjacent pore bodies radii. Indeed, by construction, the length 
of a throat $l_{i, j}$ is expressed as

$$
l_{i, j}=a-r_{p i, j}-r_{p i+1, j}
$$

where $a$ is the lattice spacing and $r_{p i, j}$ and $r_{p i+1, j}$ are the radii of the two adjacent pore bodies. As a result, the $Q_{n}\left(P_{c}\right)$ and $P_{c}(S)$ curves depend not only on the TSD but also on the PSD. Thus, the pore body size should be kept as a variable in the optimization process in order to keep generality. However, as already mentioned, the drainage process is essentially sensitive to the TSD and the impact of the PSD on the flow rate can be reasonably neglected. Thus, the pore body size is taken as constant (equal to $80 \mu \mathrm{m}$ ) in what follows. Consequently, generation of individuals is carried out by only varying the throat sizes. The minimum and maximum radii of the TSD are determined from the invasion percolation procedure carried out on the reference network. The minimum throat radius, $r_{\min 0}$, is given by Eq. (2) at the corresponding pressure step when the saturation becomes equal to zero. Conversely, the maximum throat radius, $r_{\max 0}$, cannot be as exactly determined as $r_{\min 0}$. It is approximately specified by multiplying by a factor 1.3 the radius corresponding to the first pressure step at which the saturation is no longer equal to 1 .

In order to introduce diversity in the initial population, individuals are generated within $N_{0 b}$ class intervals. The $i$ th interval has also a uniform p.d.f. with minimum and maximum radii, $r_{\min } i$ and $r_{\max } i$, respectively, given by $r_{\min i}=\frac{\left(N_{0 b}+i\right) r_{\min 0}+\left(N_{0 b}-i\right) r_{\max } 0}{2 N_{0 b}}$ and $r_{\max } i=$ $\frac{\left(N_{0 b}-i\right) r_{\min 0}+\left(N_{0 b}+i\right) r_{\max } 0}{2 N_{0 b}}$. The number of individuals generated in the $i$ th interval is $N_{0 i}$ so that $N_{0}=\sum_{i=1}^{N_{0 b}} N_{0 i}$. Here, $N_{0 b}=4$ and $N_{0 i}=4$. The normalized nonwetting fluid flow rate and capillary pressure retention curves, together with the corresponding fitness function of interest, are computed for each individual parent. After this initialization procedure, the genetic algorithm is executed according to the following steps.

(1) The natural selection is applied following a tournament method [26]. This is achieved by randomly choosing a subset of $N_{1}$ individuals among the generation of individuals (here, $N_{1}=5$ ) and the two ones featuring the best fitness values are selected.

(2) Two evolutionary operators, namely, crossover and mutation, are applied to the two selected parent individuals. Crossover is the process by which the two selected parent individuals give birth to a child realization by randomly exchanging throat sizes (genes). Mutation is an operation applied separately to both selected parent realizations. It consists in altering genes (the sizes of randomly chosen throats). Mutations are generally supposed to happen rarely. In the present case, the mutation rate is taken equal to 0.01 . In our case this means that in a $50 \times 50$ network, for instance, 12 throats are selected randomly in each direction. Then the sizes of these 24 throats are specified randomly between $r_{\min }$ and $r_{\text {max }}$ according a uniform p.d.f.

(3) The $Q_{n}\left(P_{c}\right)$ and $P_{c}(S)$ curves and the fitness values for the two children are computed and, among the four individuals (the two parents and the two children), the two individuals having the best fitness values are selected. They are kept to form the new generation.
(4) Steps 1-3 are repeated until there are no more individuals from the current parent population and a new generation of $N_{0}$ individuals is formed.

(5) The process made of steps 1-4 is iterated until convergence is reached. This means that the generation of $N_{0}$ networks obtained at the end of steps 1-4 forms the new parent generation when convergence has not been reached. By convergence, it is meant that the fitness of all the individuals from one generation to the following one does not change by more than about $0.1 \%$.

For all the cases under study in this paper, 200 generations (iterations) were enough to reach convergence at which the solution is estimated to be close to the global solution. Then a new step is performed using the hill climbing algorithm.

The solution obtained from the genetic algorithm is global in the sense that the population of all the individuals is optimized by adjusting their overall genes content. When considering a network with its given TSD, the genetic algorithm, which operates on randomly selected throats, keeps in fact this TSD. A network with a Gaussian TSD, for example, is such that throat radii close to the mean radius are more likely to be selected than others. This limits the population diversity. The objective of the hill climbing algorithm, which, like the genetic algorithm, is a searching algorithm, is to gradually modify the throat size p.d.f. within a single individual so as to converge toward the TSD of the reference network. To this purpose, the hill climbing algorithm is operated on the best fitted individual in the population once the genetic algorithm ends. It attempts to find a better solution by adjusting only a single variable among all the variables. By contrast to the genetic algorithm where the throat radii are adjusted, the hill climbing algorithm [25] aims at the adjustment of the throat densities. Once the lower and upper bounds of the TSD are specified at the starting point of the optimization process, the distribution can be divided into class intervals, or bins. Then the density of throat sizes belonging to each bin can be determined, i.e., the fraction of throats having a size within the radius interval corresponding to the bin. The hill climbing algorithm aims at determining the optimal density of each bin. To summarize, the genetic algorithm is a multidirectional search algorithm operating on radii belonging to different distribution bins, whereas the hill climbing algorithm is a unidirectional algorithm that operates only on radii belonging to a pair of defined bins. Actually, as the sum of all distribution bin densities must be equal to 1 , the hill climbing algorithm operates on two bin densities by increasing one (the positive bin) and decreasing the other (the negative bin) with the same amount. Thus, two bins must be selected. In our case, the two bins of the best fitted individual resulting from the genetic algorithm are randomly chosen. A relatively small randomly chosen number of throats are interchanged between the negative and positive bins. If the fitness function is improved, the modified individual is retained and the hill climbing process is repeated until the fitness function does not improve. When it is not improved, another test is performed by randomly choosing a different couple of bins. The fitness function for the hill climbing algorithm is equivalent to the one considered for the genetic algorithm and is denoted by fit(d) in its generic form, where $\mathbf{d}$ is the distribution density vector. The difference between the genetic and hill climbing algorithms lies in the 


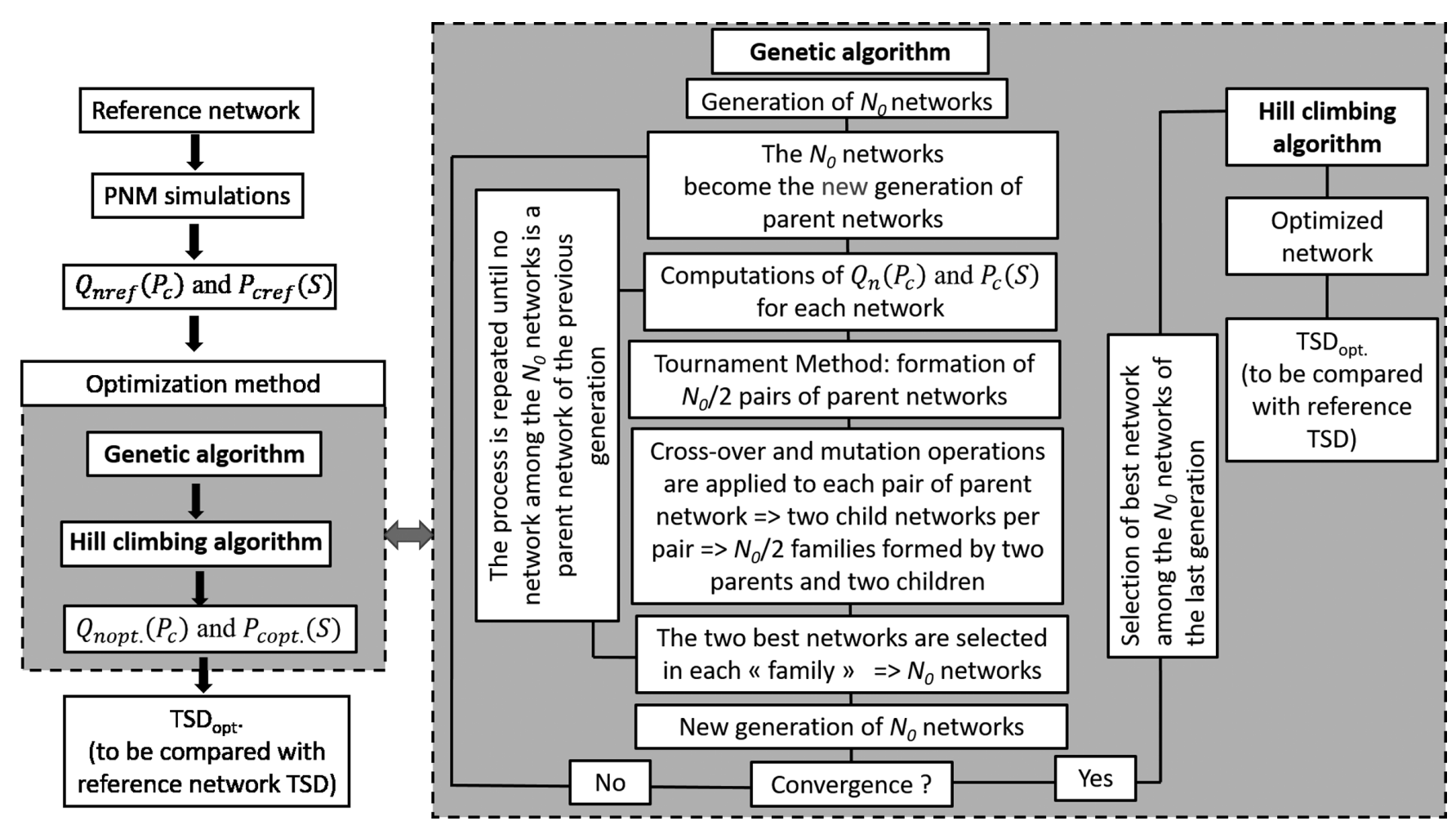

FIG. 6. Optimization algorithm chart.

variables to control for minimizing the objective function. Fitness functions are detailed in the following section. The optimization algorithm is summarized in the chart depicted in Fig. 6.

\section{B. Fitness functions}

The direct problem solution provides the $Q_{n}\left(P_{c}\right)$ and $P_{c}(S)$ curves of the reference network, i.e., the one for which the TSD from either the normalized flow rate curve or the capillary pressure curve data, or both, is to be determined. As indicated above, the network is a $2 \mathrm{D}, 50 \times 50$ square network, with a lattice spacing of $350 \mu \mathrm{m}$ and a chosen TSD as will be further presented in the results section. The initial $N_{0 i}$ individuals (also 2D, $50 \times 50$ square networks with a lattice spacing of $350 \mu \mathrm{m}$ ) are generated in the $N_{0 b}$ class intervals having a uniform TSD with their own minimum and maximum radii as mentioned above.

For the same pressure steps as the ones considered for the reference $Q_{n}\left(P_{c}\right)$ and $P_{c}(S)$ curves, the optimization process, following the genetic and hill climbing algorithms, is performed. Again, this implies to solve the direct problem for every individual considered in the optimization process. The TSD at each step of the optimization procedure is compared to the reference one by evaluating the fitness function, which is defined as the cumulative error function. If the optimization is based on the normalized flow rate $Q_{n}=\frac{Q_{\mathrm{NW}}}{Q_{\max }}$, this fitness function is given by

$$
\mathrm{fit}_{Q_{n}}(\boldsymbol{\omega})={ }_{j=1}^{n}\left|Q_{n-\mathrm{ref} j}-Q_{n-\text { ind } j}(\boldsymbol{\omega})\right|, \quad \boldsymbol{\omega}=\boldsymbol{r}_{t}, \boldsymbol{d}
$$

where $n$ is the total number of pressure steps; $Q_{n-\text { ref } j}$ and $Q_{n-\text { ind } j}$ are, respectively, the reference and individual normalized flow rates corresponding to the $j$ th pressure increment; and $\boldsymbol{\omega}=\boldsymbol{r}_{t}, \boldsymbol{d}$ depending on whether the algorithm is at the genetic or hill climbing stage. Again, flow rates are considered so as to use the same convention as for the FFDP [10]. However, it should be clear that $Q_{n}=k_{r}$ in the quasistatic limit under consideration, where $k_{r}$ is the nonwetting fluid relative permeability.

Similarly, if the optimization is based on the capillary pressure curve, the fitness function is defined as

$$
\mathrm{fit}_{\varepsilon P_{c}}(\boldsymbol{\omega})=\varepsilon_{j=1}^{n}\left|\varepsilon_{\mathrm{ref}} S_{\text {ref } j}-\varepsilon_{\text {ind }} S_{\text {ind } j}(\boldsymbol{\omega})\right|, \quad \boldsymbol{\omega}=\boldsymbol{r}_{t}, \boldsymbol{d}
$$

where $S_{\text {ref } j}$ and $S_{\text {ind } j}$ are the reference and individual saturations at the $j$ th pressure increment, respectively. As can be inferred from Eq. (22), where $\varepsilon_{\text {ref }}$ and $\varepsilon_{\text {ind }}$ are, respectively, the reference and individual porosities, the porosity is actually also considered as a variable to optimize. By definition, $\varepsilon S$ is computed as

$$
\varepsilon S=\frac{V_{\text {noninv }}}{V_{\text {total }}}
$$

where $V_{\text {noninv }}$ is the volume of the pore space not invaded at the $j$ th step and $V_{\text {total }}$ is the total volume of the porous medium. The wetting fluid volume fraction, $\varepsilon S$, does not involve the total pore space volume, $V_{\text {pores }}$, but only the volume of the noninvaded pore bodies and throats as a variable to adjust. This is preferable to the saturation as the latter depends on both $V_{\text {noninv }}$ and $V_{\text {pores }}$ that can have different impact on the fitness function. Actually, the variable that is adjusted is the 


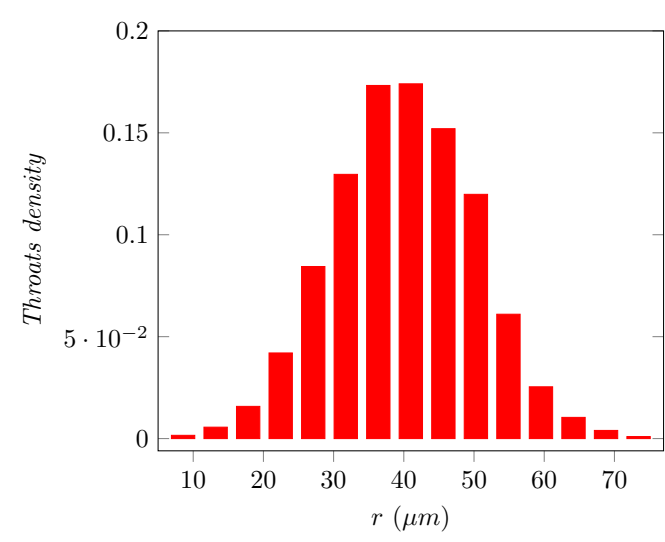

(a)

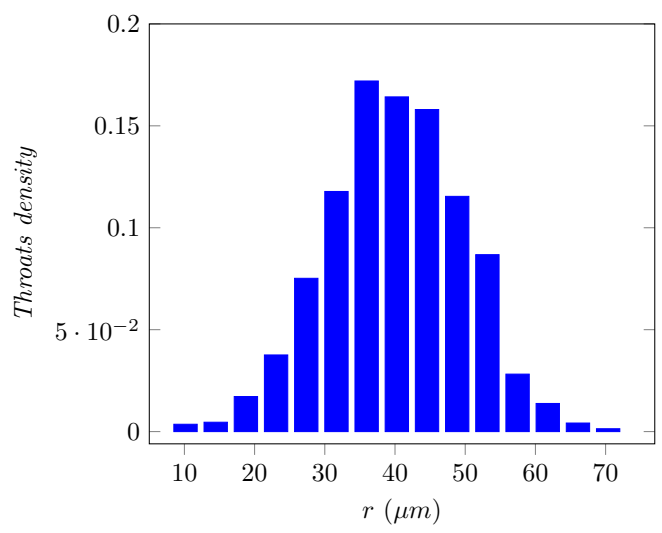

(c)

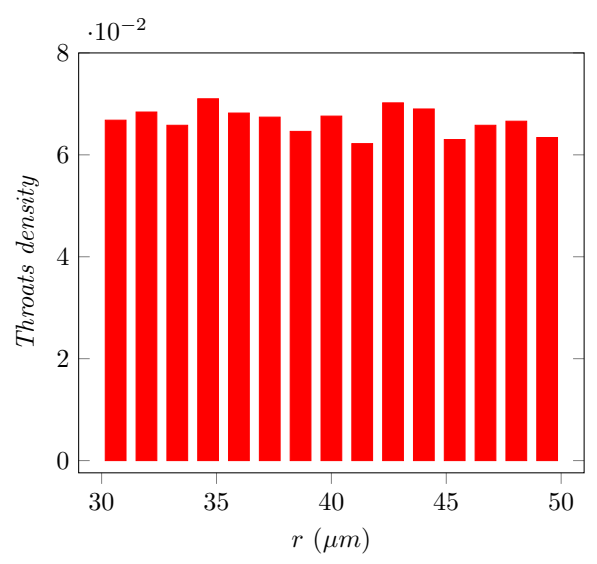

(b)

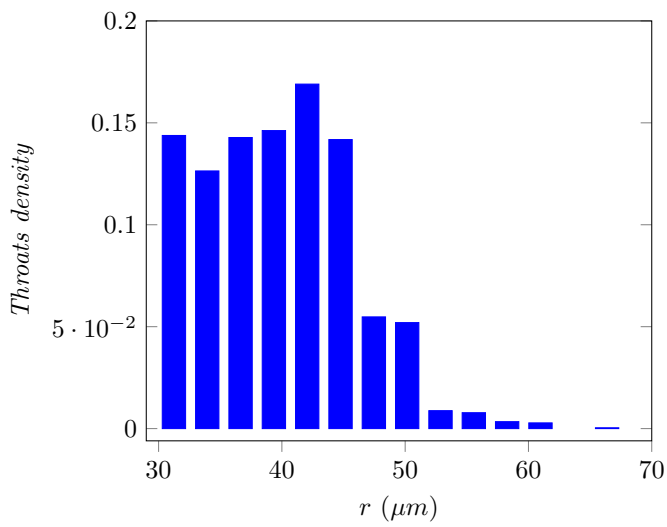

(d)

FIG. 7. Top: Reference Gaussian TSD (a) and reference uniform TSD (b) for a $50 \times 50$ square network. Bottom: TSD obtained from the optimization procedure using both the normalized flow rate and capillary pressure curve as target data. The TSDs in (c) and (d) are to be compared with the TSDs in (a) and (b), respectively.

porosity when the saturation is equal to 1 ; otherwise, it is the saturation function.

When both the flow rate and the capillary pressure curves are considered, then the fitness function is defined as

$$
\begin{aligned}
\mathrm{fit}_{Q_{n}-\varepsilon P_{c}}(\boldsymbol{\omega})= & { }_{j=1}^{n}\left|Q_{n-\operatorname{ref} j}-Q_{n-\mathrm{ind} j}(\boldsymbol{\omega})\right| \\
& +f_{p}^{n}\left|\varepsilon_{\text {ref }} S_{\text {ref } j}-\varepsilon_{\text {ind } k} S_{\text {ind } j}(\boldsymbol{\omega})\right|, \\
\boldsymbol{\omega} & =\boldsymbol{r}_{t}, \boldsymbol{d}
\end{aligned}
$$

where $f_{p}$ is a penalization factor that is taken as constant and computed as

$$
f_{p}=\frac{1}{\varepsilon_{\mathrm{ref}}} .
$$

The idea with the weighted definition in Eq. (24) is actually to give more importance to the capillary pressure curve in the optimization process.

\section{Results}

Results are first discussed for a reference network with a Gaussian TSD having a mean radius of $40 \mu \mathrm{m}$ and a standard deviation of $10 \mu \mathrm{m}$. This reference TSD is shown in Fig. 7(a). As mentioned in Sec. VIB, the initial population that must be generated at the beginning of the optimization process is composed of realizations with uniform distributions, thus with a type of p.d.f. different from the Gaussian one of the reference network.

Results on optimized TSDs when the target data are only the capillary pressure curve [Eq. (22)] (left column), only the normalized flow rate curve [Eq. (21)] (middle column), and both the retention and normalized flow rate curves [Eq. (24)] (right column) are represented in Fig. 8. Corresponding quantitative results are reported in Table II.

As can be seen from Fig. 8 and Table II, using the capillary pressure leads to a better result than when only the normalized flow rate curve is employed. Not surprisingly, the best result is obtained when both the retention curve and the normalized flow rate curve are used in the optimization process. Indeed, comparing the reference TSD in Fig. 7(a) to the optimized one obtained with this procedure in Fig. 7(c) shows a very good agreement. This is also confirmed by the results in Table II. As illustrated in Fig. 8 (right column), the retention and normalized flow rate curves resulting from the optimization procedure reproduce very well the corresponding curves of the reference case. 

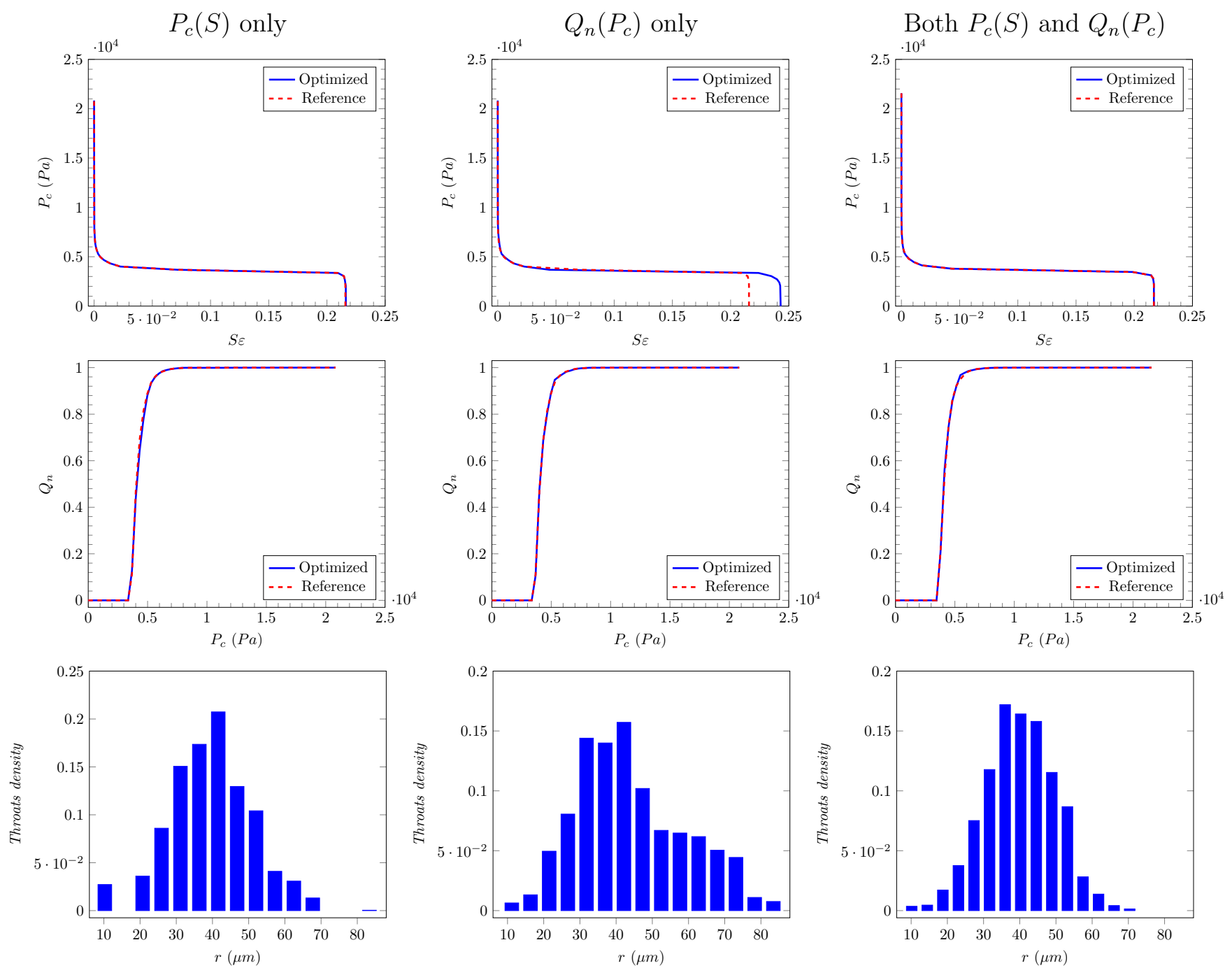

FIG. 8. Results of optimization procedure considering the capillary pressure curve only as target data (left column), the normalized flow rate curve only (middle column), and both the normalized flow rate (equivalent to $k_{r}$ ) and capillary pressure curves (right column), when the reference p.d.f. is Gaussian [as depicted in Fig. 7(a)].

A similar test was performed when the reference TSD is uniform [Fig. 7(b)]. The results are represented in Fig. 8 and quantitative data in Table III. Here again, the TSD obtained considering the capillary pressure curve only is closer to the reference one than when the optimization is based on the normalized flow rate curve only. The best result is again obtained when both the capillary pressure and the normalized flow rate curves are used in the optimization procedure. However, as illustrated in Figs. 7(b) and 7(d), the optimization is less performant than in the case of a Gaussian TSD for the reference medium. This discrepancy can be explained by the uncertainty on the distribution upper bound associated to the difficulty of accurately determining the maximum throat radius, $r_{\max 0}$, as mentioned in Sec. VIA. This inaccuracy leads us to introduce some throats greater than the maximum radius $(50 \mu \mathrm{m})$ in the reference distribution during the optimization procedure.

TABLE II. Comparison between the reference and optimized TSDs when the reference TSD is the Gaussian TSD depicted in Fig. 7(a).

\begin{tabular}{|c|c|c|c|c|c|c|c|}
\hline \multirow[b]{2}{*}{$\begin{array}{l}\text { Statistical } \\
\text { moments }(\mu \mathrm{m})\end{array}$} & \multirow[b]{2}{*}{$\begin{array}{l}\text { Reference } \\
\text { TSD }\end{array}$} & \multicolumn{2}{|c|}{$P_{c}(S)$ only } & \multicolumn{2}{|c|}{$Q_{n}\left(P_{c}\right)$ only } & \multicolumn{2}{|c|}{ Both $P_{c}(S)$ and $Q_{n}\left(P_{c}\right)$} \\
\hline & & $\begin{array}{l}\text { Optimized } \\
\text { TSD }\end{array}$ & $\begin{array}{l}\text { Relative } \\
\text { error }(\%)\end{array}$ & $\begin{array}{l}\text { Optimized } \\
\text { TSD }\end{array}$ & $\begin{array}{l}\text { Relative } \\
\text { error }(\%)\end{array}$ & $\begin{array}{l}\text { Optimized } \\
\text { TSD }\end{array}$ & $\begin{array}{l}\text { Relative } \\
\text { error }(\%)\end{array}$ \\
\hline Mean & 40 & 39.57 & $1.08 \%$ & 43.53 & 8.83 & 40.24 & 0.6 \\
\hline Standard deviation & 9.9 & 11.37 & 14.85 & 15.18 & 53.33 & 9.77 & 1.31 \\
\hline Skewness & -0.01 & 0.06 & & 0.45 & & 0.043 & \\
\hline Kurtosis & 3 & 3.275 & 9.16 & 2.57 & 14.33 & 2.77 & 7.67 \\
\hline
\end{tabular}


TABLE III. Comparison between the reference TSD and the obtained TSD, where the reference TSD is the uniform TSD depicted in Fig. 7(b).

\begin{tabular}{|c|c|c|c|c|c|c|c|}
\hline \multirow[b]{2}{*}{$\begin{array}{l}\text { Statistical } \\
\text { moments }(\mu \mathrm{m})\end{array}$} & \multirow[b]{2}{*}{$\begin{array}{c}\text { Reference } \\
\text { TSD }\end{array}$} & \multicolumn{2}{|c|}{$P_{c}(S)$ only } & \multicolumn{2}{|c|}{$Q_{n}\left(P_{c}\right)$ only } & \multicolumn{2}{|c|}{ Both $P_{c}(S)$ and $Q_{n}\left(P_{c}\right)$} \\
\hline & & $\begin{array}{l}\text { Optimized } \\
\text { TSD }\end{array}$ & $\begin{array}{l}\text { Relative } \\
\text { error }(\%)\end{array}$ & $\begin{array}{l}\text { Optimized } \\
\text { TSD }\end{array}$ & $\begin{array}{l}\text { Relative } \\
\text { error }(\%)\end{array}$ & $\begin{array}{c}\text { Optimize TSD } \\
\text { TSD }\end{array}$ & $\begin{array}{l}\text { Relative } \\
\text { error }(\%)\end{array}$ \\
\hline Mean & 40 & 39.88 & $0.29 \%$ & 43.67 & 9.18 & 39.93 & 0.18 \\
\hline Standard deviation & 5.77 & 6.36 & 10.22 & 10.72 & 85.79 & 5.97 & 3.47 \\
\hline Skewness & 0.01 & 0.91 & & 0.97 & & 0.42 & \\
\hline Kurtosis & 1.8 & 5.06 & 181 & 2.99 & 66.1 & 2.93 & 62.78 \\
\hline
\end{tabular}

This effect is of course much less sensitive in the case of distributions of Gaussian or log-normal types for which the relative density of throats close to the distribution upper bound is much lower than in a uniform distribution.

As also shown in Fig. 9, the capillary and normalized flow rate curves of the optimized network almost perfectly match the ones of the reference network. However, the match for the capillary curve is not satisfactory for the lower values of the capillary pressure when only the normalized flow rate data are used in the optimization procedure. This also holds in the case of the Gaussian reference TSD (see Fig. 8). This is an additional illustration of the impact of the distribution upper bound specification and also of the percolation threshold issue discussed in Sec. V.
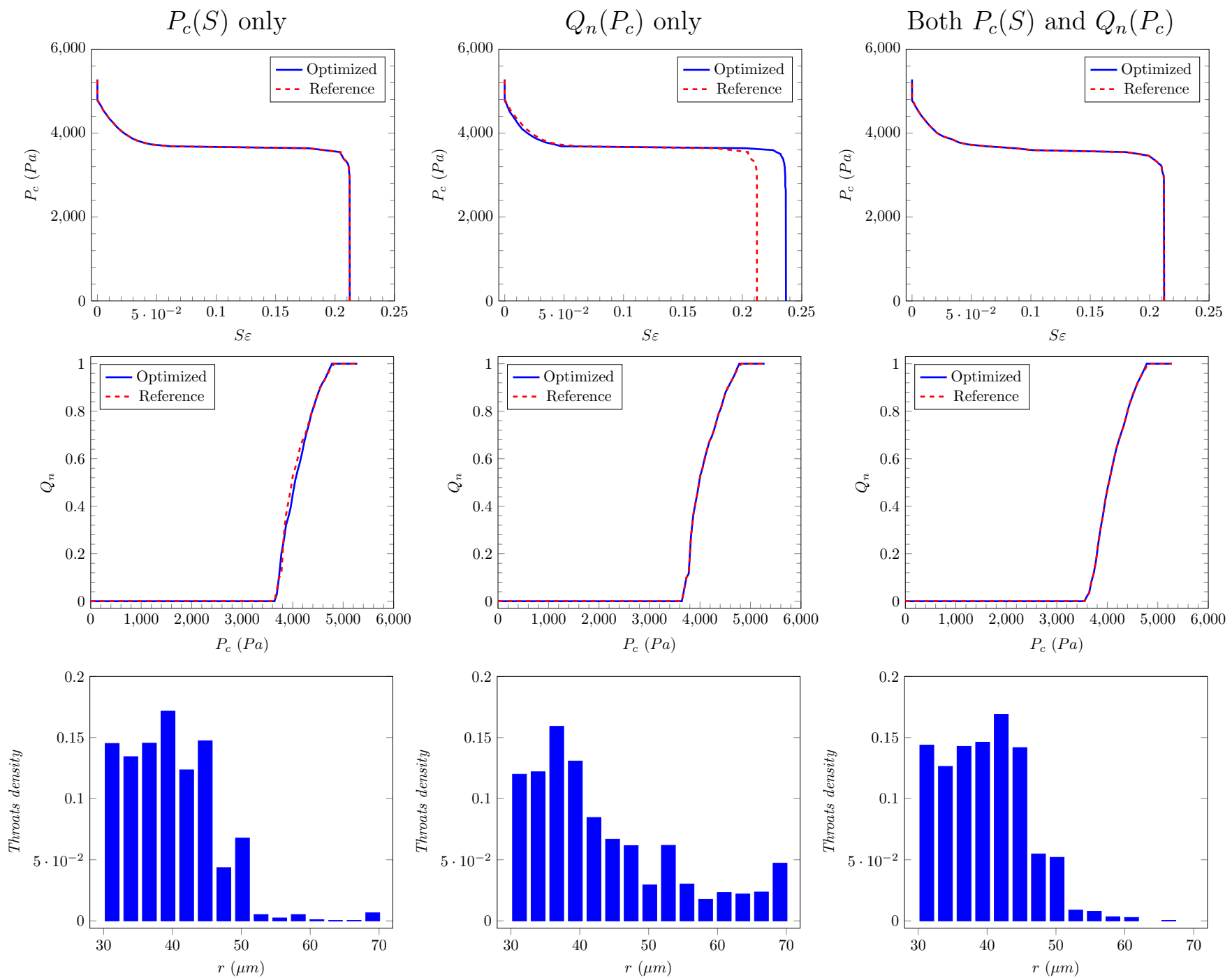

FIG. 9. Optimization results considering only the capillary pressure curve as target data (left column), only the normalized flow rate curve (middle column), and both the normalized flow rate and capillary pressure curves (right column). The reference p.d.f. is the uniform one reported in Fig. 7(b). 


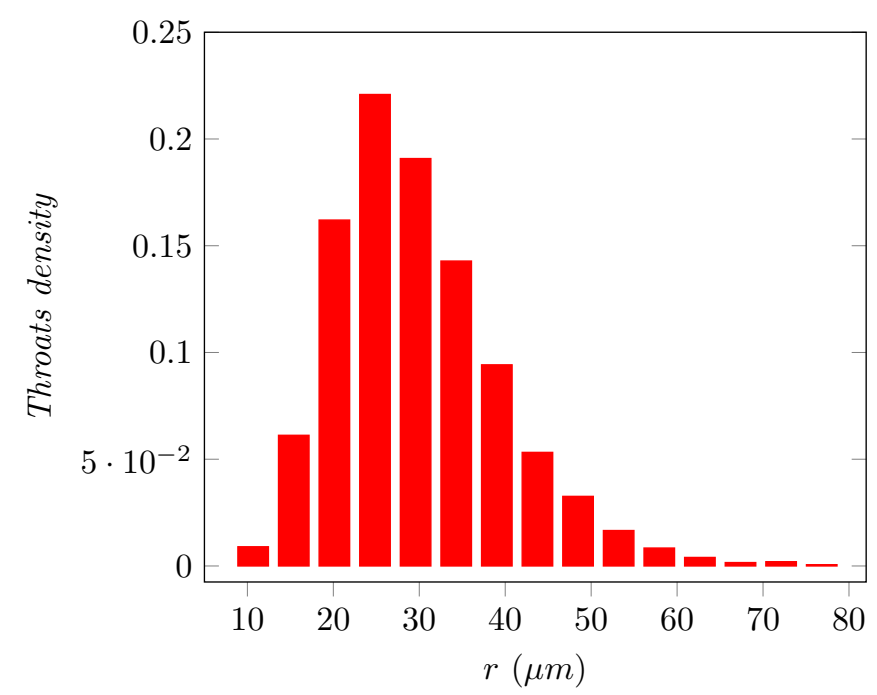

(a)

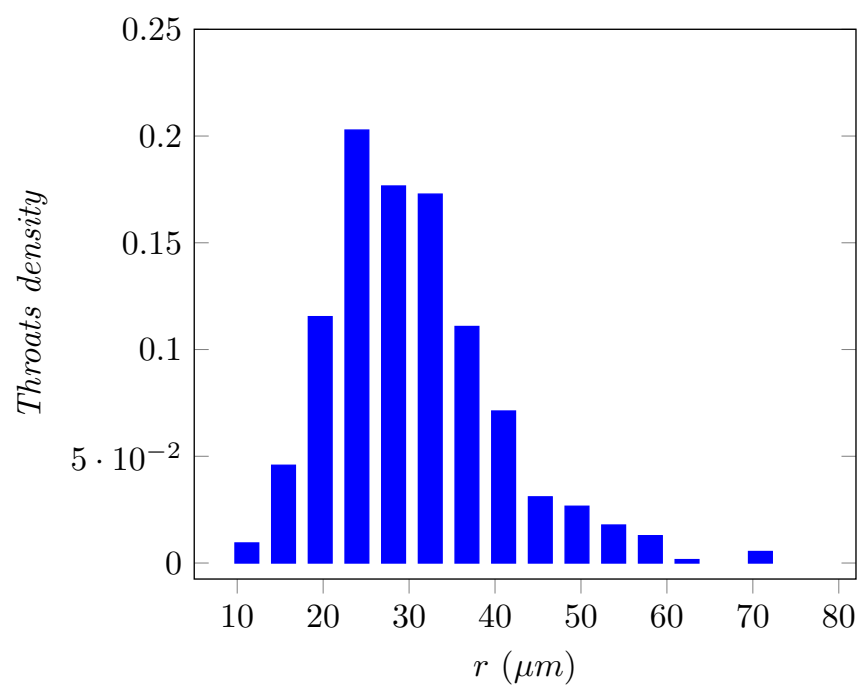

(c)

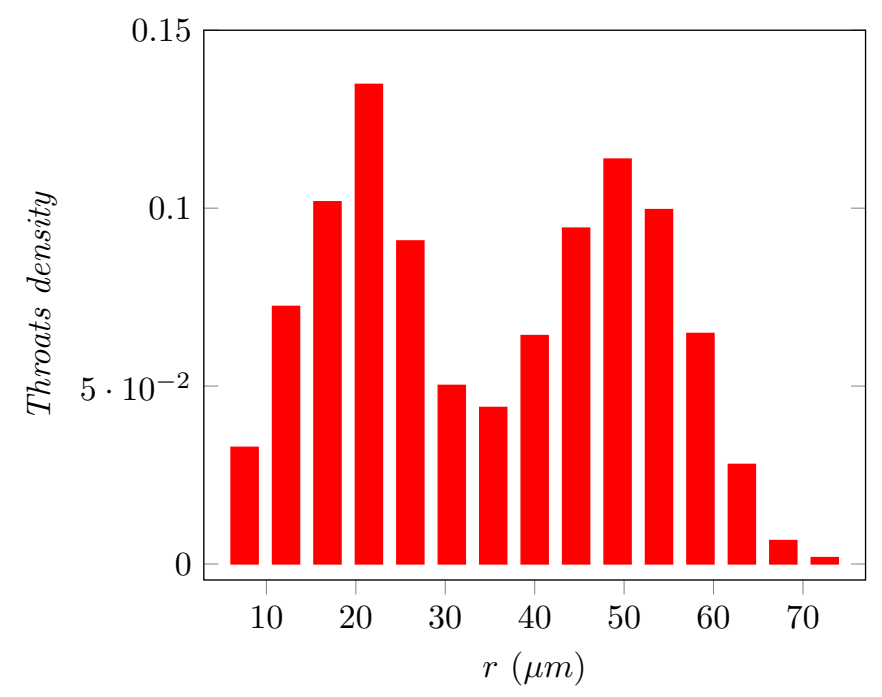

(b)

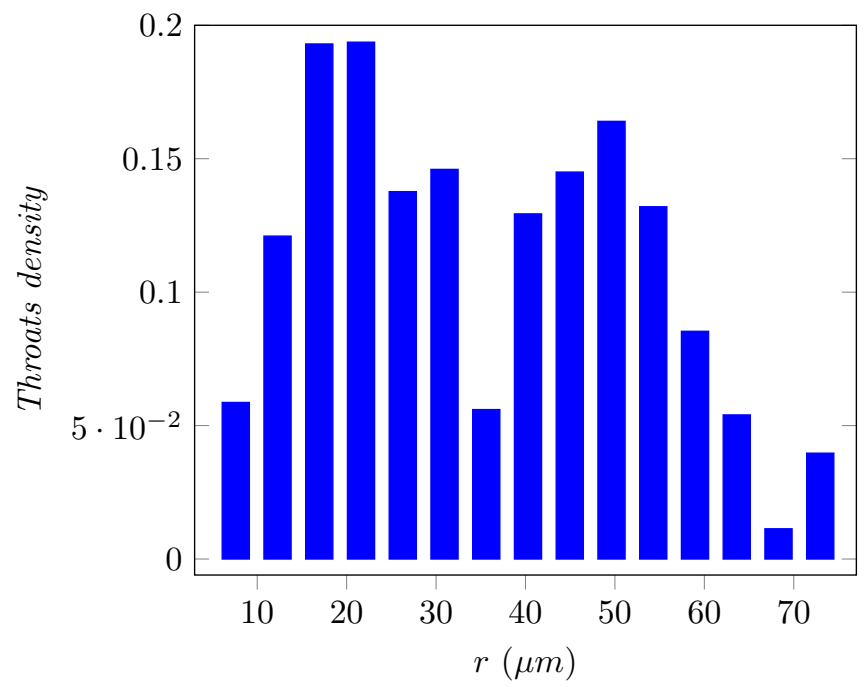

(d)

FIG. 10. Results of the optimization procedure using both the normalized flow rate and capillary pressure curves as target data for a $50 \times 50$ square network having a reference log-normal TSD (a) and bimodal TSD (b). Figures in the left and right columns are for the log-normal and bimodal distributions, respectively. The optimized TSDs in (c) and (d) are to be compared with the reference TSDs in (a) and (b), respectively.

Two additional tests were performed considering a reference log-normal distribution [as depicted in Fig. 10(a)] and the expected more challenging bimodal distribution depicted in Fig. 10(b). For these cases, only the full optimization proce- dure, i.e., the one based on both $P_{c}(S)$ and $Q_{n}\left(P_{c}\right)$, was used. As can be seen from Fig. 10 and Table IV, results are quite satisfactory. Here also, as shown in Fig. 11, the match between the reference capillary pressure and the normalized flow rate

TABLE IV. Comparison between the reference and optimized TSDs using both the capillary pressure and normalized flow rate curves as target data, where the reference TSD is the log-normal one depicted in Fig. 10(a) or the bimodal one depicted in Fig. 10(b).

\begin{tabular}{|c|c|c|c|c|c|c|}
\hline \multirow[b]{2}{*}{ Statistical moments $(\mu \mathrm{m})$} & \multicolumn{3}{|c|}{ Log-normal TSD } & \multicolumn{3}{|c|}{ Bimodal TSD } \\
\hline & Reference TSD & Optimized TSD & Relative error $(\%)$ & Reference TSD & Optimized TSD & Relative error $(\%)$ \\
\hline Mean & 39.84 & 39.85 & 0.003 & 35.3 & 35.09 & 0.6 \\
\hline Standard deviation & 9.83 & 9.8 & 0.3 & 16.61 & 17.07 & 2.8 \\
\hline Skewness & 0.63 & 0.58 & 7.94 & 0.06 & 0.245 & \\
\hline Kurtosis & 3.48 & 3.33 & 4.31 & 1.71 & 2.01 & 17.54 \\
\hline
\end{tabular}




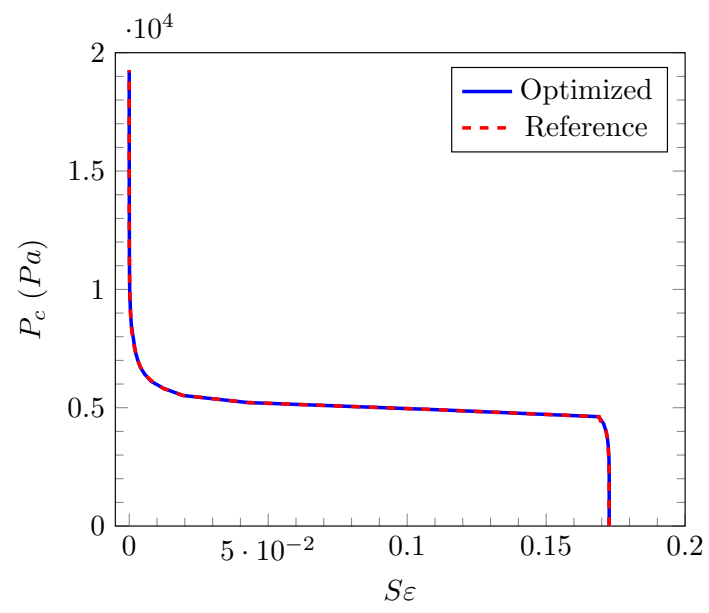

(a)

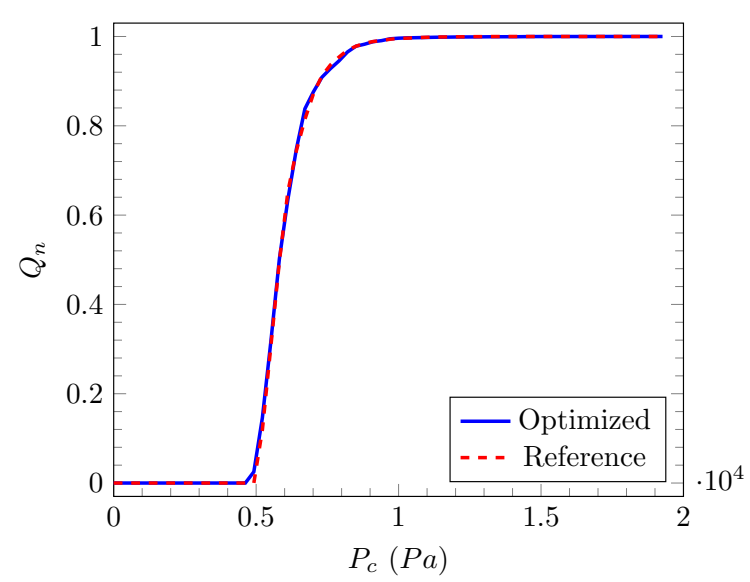

(c)

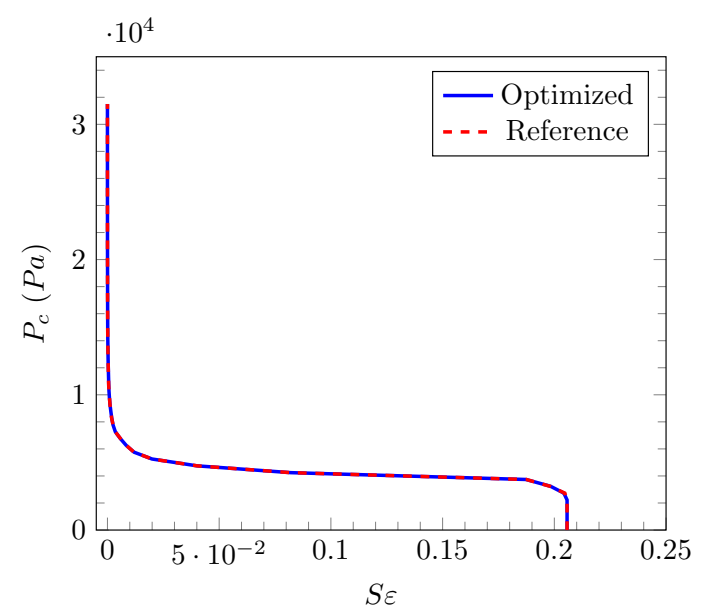

(b)

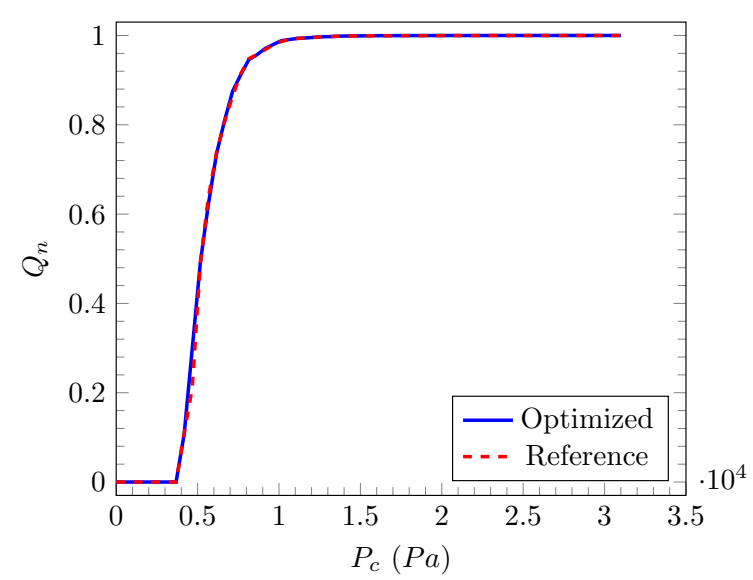

(d)

FIG. 11. Results of the optimization procedure using both the normalized flow rate and capillary pressure curves as target data for a $50 \times 50$ square network having a reference log-normal TSD (a) and bimodal TSD (b). Figures in the left and right columns are for the log-normal and bimodal distributions, respectively. Comparison of the reference and optimized capillary (or retention) curves (a), (b) and normalized flow rate (or relative permeability) curves (c), (d).

curves and the curves resulting from the optimization procedure is excellent. Finally, among the four reference TSDs considered here, results obtained for the uniform one are the less accurate, mainly because of the uncertainty associated with the distribution upper bound specification.

\section{DISCUSSION}

To further illustrate the interest of the optimization approach compared to the conventional approaches based on the consideration of the parallel tubes model and either the normalized flow rate curve [Eq. (13)] or the Young-Laplace equation and the retention curve [Eq. (6)], the simple model (i.e., the parallel tubes model) was applied to the bimodal reference network [Fig. 10(b)]. The results are represented in Fig. 12 and reported in Table V.

As can be seen, the bimodal nature of the distribution cannot be identified using the simple model. The approach based on the normalized flow rate curve and the parallel tubes model, i.e., Eq. (13), only captures the throat sizes below the percolation threshold (as discussed in Sec. V). As a result, only throats in the range of the lower peak distribution are retrieved. When the capillary pressure curve combined with the Young-Laplace equation is used, i.e., Eq. (6), throats over a larger range of size are identified but the bimodal nature of the distribution is not at all retrieved. However, as indicated in Table $\mathrm{V}$, the procedure based on the capillary pressure curve, i.e., Eq. (6), leads to a satisfactory estimate of the throat size mean value. Actually, as can be seen also from Table I, the use of the capillary pressure curve information in conjunction with the Young-Laplace equation for the parallel tubes model leads to the same result, i.e., the throat size mean value is reasonably well predicted. Note from Tables II and III that it is also the case with the optimization procedure: the throat size mean value is well predicted when the capillary pressure curve information is used [and also when both the $P_{c}(S)$ and $Q_{n}\left(P_{c}\right)$ information are used as can be seen from Tables II-V]. An explanation of this fact, i.e., the fact that the use of the retention curve in conjunction with the Young-Laplace equation and the parallel tubes model leads to a good estimate of the mean throat size, is proposed in the Appendix. 


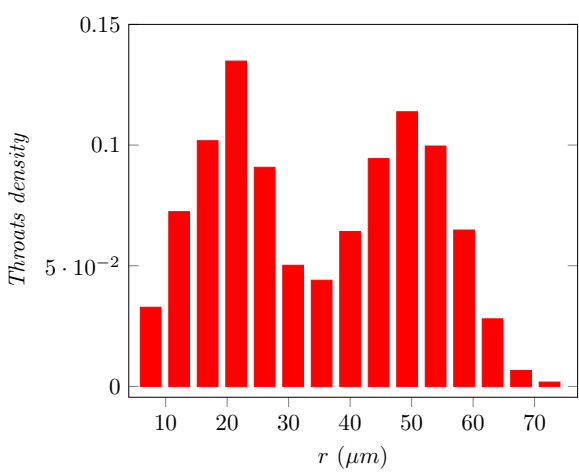

(a)

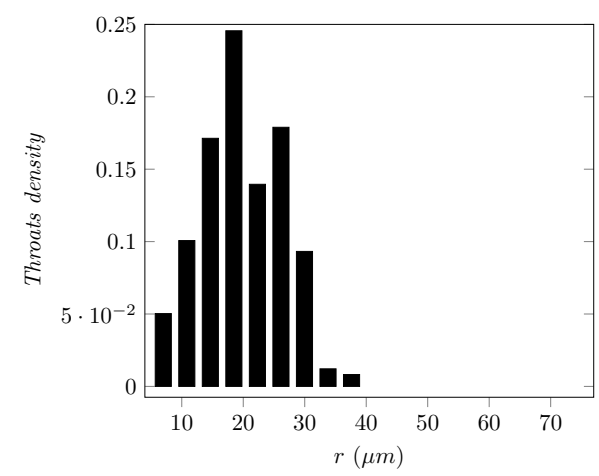

(b)

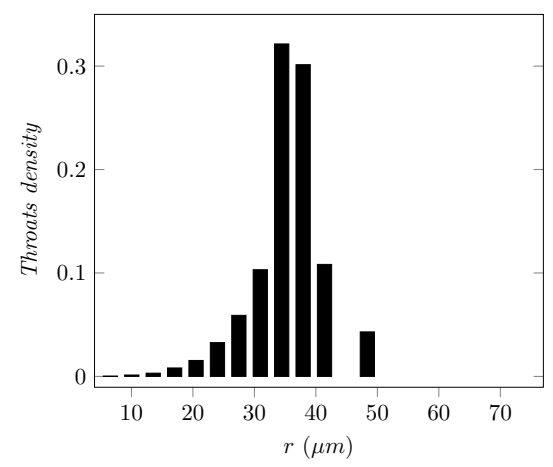

(c)

FIG. 12. Reference bimodal TSD (a), SMTSD identified using Eq. (13), i.e., the normalized flow rate curve and the parallel tubes model (b), and SMTSD identified using Eq. (6), i.e., the capillary pressure curve and the parallel tubes model (c).

For perspective, we offer the following remarks on the above results. Although the TSD identification based on the optimization procedure clearly leads to much better results than the simple parallel tubes model, several points are worth being recalled or discussed. First, trapping phenomena were ignored. It would be interesting to investigate the possible impact of this phenomenon on the TSD determination. Second, the optimization procedure has been tested considering only the throat size variability. In other words, the pore body size variability (the PSD) was not considered. In this respect, it would be interesting to explore the ability of the optimization procedure to capture both the PSD and the TSD. Third, the optimization procedure has been performed for given pore network structures, i.e., square or cubic in our simulations. It can certainly be argued that, in general, the network structure is unknown a priori. In addition, the pore network that can represent a real porous medium is rarely structured but rather unstructured. This means that the distance between two adjacent pore bodies may vary, as well as the local coordination number, i.e., the number of pore bodies to which a given pore body is directly connected. Therefore, an interesting question to address will be to test the impact of the structure. In other words, if cubic networks are used, for instance, in the optimization procedure with reference data obtained from a medium the 3D pore network of which is obviously not cubic, will the procedure still be relevant, or at least provide significantly better results than those obtained using the much simpler procedures making use of Eq. (6) or Eq. (13)? Since the microstructures of porous media can be quite diverse, it can be surmised that the quality of the results will also be quite diverse depending on the medium pore network structure and how close it is from a cubic network. In principle, the optimization procedure can of course be used for pore networks different from cubic. However, this may be cumbersome, keeping in mind that many realizations of the network must be generated. Here, the use of stochastic network generators [28] might be an option. From a practical point of view, a simpler option may be to create a catalog of various pore network structures (cubic, Voronoi, etc.) and choose the one which seems the closest to the structure of the porous medium to be characterized. This choice could be guided by images from the microstructure or partial information on this structure. However, when detailed digital 3D images of the microstructure are available, it is probably simpler to try to extract the PSD and/or the TSD directly from the images $[8,9]$. Nevertheless, it would be interesting to test whether the use of the $Q_{n}\left(P_{c}\right)$ and $P_{c}(S)$ curves with the optimization procedure might help characterize the microstructure by testing different pore network structures available in the catalog. Another type of situation is when the throat or pore body sizes change due to some coupled phenomena (particle trapping, dissolution or precipitation, or biofilm development). Using the optimization procedure to characterize the pore network evolution can be much simpler than repeated 3D imaging. In this case, one could use a stochastic pore network generator based on data obtained on initial images of the microstructure. Nevertheless, this requires determining the retention curves and the nonwetting fluid normalized flow rate (as well as the porosity and the permeability) repeatedly. Finally, the network size considered in this paper remains quite small, and this was mainly motivated by the interest which was focused on the characterization of thin porous media with only few pores over their thickness [29]. One can wonder whether increasing the network size might affect the results. Although tests in this

TABLE V. Comparison of the throat size mean values of the reference TSD with those obtained using the parallel tubes model and normalized flow rate curve, Eq. (13), or the capillary pressure curve combined with the Young-Laplace equation, Eq. (6), for the reference bimodal TSD depicted in Fig. 10(b).

\begin{tabular}{|c|c|c|c|c|}
\hline \multirow{3}{*}{$\frac{\text { Reference TSD }}{\text { Mean }(\mu \mathrm{m})}$} & \multicolumn{4}{|c|}{ Parallel tubes model } \\
\hline & \multicolumn{2}{|c|}{$Q_{n}\left(P_{c}\right)$} & \multicolumn{2}{|c|}{$P_{c}(S)$} \\
\hline & Mean $(\mu \mathrm{m})$ & Relative error $(\%)$ & Mean $(\mu \mathrm{m})$ & Relative error $(\%)$ \\
\hline 35.3 & 19.6 & 44.48 & 35.3 & 0.3 \\
\hline
\end{tabular}


direction could be interesting, it can be noted, for instance, that the problem associated with the network percolation threshold, when the flow rate information is used, will still be present whatever the network size. As a consequence, the simple models [based on Eq. (6) or Eq. (13)] are not expected to perform better whatever the network size.

Another issue not addressed in the present paper is the possible impact of finite-size effects (see, e.g., [30]), when considering relatively small networks. The finite-size effects are of course more important in small networks and might hamper the simulations. It is assumed that the network considered in the present paper is sufficiently large for the results to be valid for much larger pore networks.

It should also be noted that the optimization procedure is significantly more intense in terms of computational resources and computer programming compared to the conventional procedures based on the parallel tubes model, which actually correspond to negligible computational times. For a $50 \times 50$ network, the computational time of one simulation corresponding to the computation of the $Q_{n}\left(P_{c}\right)$ and $P_{c}(S)$ curves considering 60 pressure steps is on average $3.66 \mathrm{~s}$ (using a single CPU 2.5-GHz Xeon processor). The genetic algorithm is run considering 200 generations. Each generation includes 16 individuals, hence involving $16 Q_{n}\left(P_{c}\right)$ and $16 P_{c}(S)$ curves simulations. Thus the computational time associated with one generation is $163.66 \approx 58.56 \mathrm{~s}$. The computational time of the genetic algorithm step is therefore $200 \times 58.56=11712 \mathrm{~s}$ ( $3 \mathrm{~h}, 16 \mathrm{~min}$ ). The hill climbing algorithm step is performed over a single network but typically requires between 500 and $700 Q_{n}\left(P_{c}\right)$ and $P_{c}(S)$ curves simulations, which correspond to a computational time of about $35 \mathrm{~min}$. The total computational time for a $50 \times 50$ network is therefore around $4 \mathrm{~h}$. Since the latter is relatively important even for a small network, the question arises as to whether the method can be realistically used in practice. In this respect, one can first notice that the code is written in MATLAB. A C or FORTRAN version would be significantly more efficient. The IP algorithm used to compute the $P_{c}(S)$ curve could be made more effective, (see for instance [31,32]). Some algorithm parallelization [a genetic algorithm, a linear system solver for the computation of the $Q_{n}\left(P_{c}\right)$ curve, etc.] is also expected to considerably reduce the computational time. Also, as mentioned previously, the porosimetry method considered in the paper is primarily developed for the characterization of thin porous media, like fuel cell porous components, [16], filtration membranes, [10], etc. The number of pores over the thickness of such media can be quite small, less than 10 for the porous component considered in [16] for instance. Thus, the network need not necessarily be large. Also, network size for the computations of properties over a representative elementary volume is typically of a few tens of pores [5]. In summary, the possibility of seriously improving the computational performances, compared to our current code, are sufficiently significant for considering that the computational performances should not be an issue for using the method in practice.

\section{CONCLUSION}

Pore network simulations were developed to assess the performance of two conventional and simple methods aiming at the determination of the TSD of a porous medium from macroscopic data such as the retention curve and/or the normalized flow rate curve. It was shown that using the normalized flow rate curve alone, which is roughly an information similar to the one used in the FFPD technique [10], is not satisfactory since all the throats greater than the critical throat corresponding to the network percolation threshold cannot be identified. On the whole, if a parallel tubes model is considered, the procedure based on the capillary pressure curve combined with the Young-Laplace equation leads to better determination of the mean radius than the procedure based on the normalized flow rate curve. However, all the invaded pores at a given pressure step in both procedures are assigned to one pore size corresponding to the specified capillary pressure regardless of their actual sizes. Thus, these simple procedures do not make any distinction between the TSD and the PSD. In order to alleviate the limitations of these conventional TSD identification methods, a procedure combining pore network simulations and an optimization technique based on a genetic algorithm and the hill climbing algorithm was developed. This algorithm leads to much better results compared to the simpler methods. Referring again to the FFPD, the paper also indicates that the knowledge of the nonwetting fluid normalized flow rate is not sufficient to determine the TSD. Using the porosity and the retention curve as additional information is necessary to obtain satisfactory results.

However, the optimization procedure is significantly more intense in terms of computational resources and computer programming compared to the conventional procedures. Efficient algorithms, programming languages, and computational procedures (parallelization) are desirable for practical use of the optimization procedure so as to perform the optimization over a sufficiently large network in a reasonably short time. Also, a somewhat simple situation has been considered to test the optimization procedure. Only the TSD was taken into account and no variability in the pore body size was envisaged. The network structure and size were the same in the reference network and the various networks generated during the optimization procedure. Certainly, more work is needed to fully explore the capability of the approach proposed in the present paper with the aim of solving the ill-posed problem consisting in identifying the TSD (and the PSD) from macroscopic data such as the normalized flow rate and capillary pressure curves. Nevertheless, results obtained here are very encouraging and suggest to further investigate the way opened by the methodology developed in this paper.

\section{ACKNOWLEDGMENT}

This research was supported by the project "PEMFCSUDOE" (Project No. SOE1/P1/E0293), which is cofinanced by the European Regional Development Fund in the framework of the Interreg Sudoe program.

\section{APPENDIX: MEAN THROAT SIZE FROM THE RETENTION CURVE}

Some explanation of the fact that the use of the retention curve in conjunction with the Young-Laplace equation always 
TABLE VI. Comparison between the mean throat radius in the TSD and the radius predicted by Eq. (A7).

\begin{tabular}{lcccc}
\hline \hline p.d.f. & $r_{M}(\mu \mathrm{m})$ & $\lambda$ & $\bar{r}$ [from Eq. (A7)] & $\bar{r}$ (from p.d.f.) $(\mu \mathrm{m})$ \\
\hline Uniform 2D (Table I) & 30.91 & 23.5 & 29.5 & 29.6 \\
Gaussian 2D (Table I) & 20.42 & 35.3 & 19.8 & 19.9 \\
Uniform 3D (Table I) & 35.85 & 15 & 33.3 & 33.32 \\
Gaussian 3D (Table I) & 21.64 & 32.5 & 20.95 & 20.98 \\
\hline \hline
\end{tabular}

lead to a satisfactory estimate of the mean throat size is proposed in this appendix.

By definition the mean throat size is given by

$$
\bar{r}=\int_{0}^{+\infty} r f_{n}(r) d r=\int_{r_{\min }}^{r_{\max }} r f_{n}(r) d r
$$

where $f_{n}(r)$ is the normalized p.d.f. According to Eq. (8),

$$
f_{n}(r)=\frac{f(r)}{\int_{r_{\min }}^{r_{\max }} f(r) d r} .
$$

Combining Eqs. (A1) and (A2) with Eq. (5) yields

$$
\bar{r}=\frac{1}{\int_{r_{\min }}^{r_{\max }} \frac{1}{V_{p}(r)} \frac{d S}{d r} d r} \int_{r_{\min }}^{r_{\max }} \frac{r}{V_{p}(r)} \frac{d S}{d r} d r .
$$

Assuming $V_{p}(r)=\pi r^{2} L$ (parallel tubes model) yields

$$
\bar{r}=\frac{1}{\int_{r_{\min }}^{r_{\max }} r^{-2} \frac{d S}{d r} d r} \int_{r_{\min }}^{r_{\max }} r^{-1} \frac{d S}{d r} d r .
$$

To relate $S$ and $r$ the Brooks and Corey representation [33] for the retention curve may be invoked, which in our case takes the form

$$
S=\left(\frac{P_{c}}{P_{M}}\right)^{-\lambda}
$$

where $P_{M}$ and $\lambda$ are two parameters that must be fitted to represent the retention curve. Introducing $r_{M}=\frac{2 \gamma}{P_{M}}$, where $r_{M}$ actually corresponds to the radius of the largest throat at the network inlet, and using Eq. (2) for $\theta=0$ leads us to express Eq. (A5) as

$$
S=\left(\frac{r_{M}}{r}\right)^{-\lambda}
$$

Substituting Eq. (A6) into Eq. (A4) leads after some calculations and the assumption that $r_{M} \approx r_{\max }$ to

$$
\bar{r} \approx \frac{(\lambda-2)}{\lambda-1} r_{M} .
$$

Fitting the values of $P_{M}$ and $\lambda$ for the networks featuring a Gaussian and uniform TSD explored in this paper (see Sec. V) and applying Eq. (A7) lead to the results presented in Table VI.

These results justify why the capillary curve contains the required information that can restitute a relevant value of the mean throat radius.
[1] E. H. D’Hollander, Water Resour. Res. 15, 107 (1979).

[2] L. R. Stingaciu, L. Weihermüller, S. Haber-Pohlmeier, S. Stapf, H. Vereecken, and A. Pohlmeier, Water Resour. Res. 46, W11510 (2010).

[3] R. Mourhatch, T. T. Tsotsis, and M. Sahimi, J. Membr. Sci. 367, 55 (2011).

[4] W. B. Krantz, A. R. Greenberg, E. Kujundzic, A. Yeo, and S. S. Hosseini, J. Membr. Sci. 438, 153 (2013).

[5] M. J. Blunt, M. D. Jackson, M. Piri, and P. H. Valvatne, Adv. in Water Res., 25, 1069 (2002).

[6] F. Plouraboué, S. Geoffroy, and M. Prat, Phys. Fluids 16, 615 (2004).

[7] F. Plouraboué, F. Flukiger, M. Prat, and P. Crispel, Phys. Rev. E 73, 036305 (2006).

[8] R. Ziel, A. Haus, and A. Tulke, J. Membr. Sci., 323, 241 (2008).

[9] H. Dong and M. J. Blunt, Phys. Rev. E 80, 036307 (2009).

[10] K. R. Morison, J. Membr. Sci. 325, 301 (2008).

[11] R. I. Peinador, J. I. Calvo, P. Prádanos, L. Palacio, and A. Hernández, J. Membr. Sci. 348, 238 (2010).

[12] C. Pan, M. Hilpert, and C. T. Miller, Water Resour. Res. 40, W01501 (2004).

[13] A. Q. Raeini, M. J. Blunt, and B. Bijeljic, Adv. Water Resour. 74, 116 (2014).
[14] V. Joekar-Niasar, S. M. Hassanizadeh, and A. Leijnse, Transp. Porous Media 74, 201 (2008).

[15] A. A. Helba, M. Sahimi, L. E. Scriven, and H. T. Davis, SPE Reservoir Eng. 7, 123 (1992).

[16] T. Agaesse, A. Lamibrac, F. Buechi, J. Pauchet, and M. Prat, J. Power Sources 331, 462 (2016).

[17] M. J. Blunt, Curr. Opin. Colloid Interface Sci. 6, 197 (2001).

[18] M. Sahimi, Flow and Transport in Porous Media and Fractured Rock: From Classical Methods to Modern Approaches (Wiley, New York, 2005).

[19] R. Lenormand, E. Touboul, and C. Zarcone, J. Fluid Mech. 189, 165 (1988).

[20] D. Wilkinson and J. F. Willemsen, J. Phys. A: Math. Gen. 16, 3365 (1983).

[21] F. Ebrahimi, Comput. Sci. Eng. 12, 84 (2010).

[22] D. Zhou, M. Blunt, and F. M. Orr, Jr., J. Colloid Interface Sci. 187, 11 (1997).

[23] D. Stauffer and A. Aharony, Introduction to Percolation Theory (Taylor \& Francis, London, 1992).

[24] C. W. Gardiner, Handbook of Stochastic Methods (Springer, New York, 1985).

[25] S. C. Su, C. J. Lin, and C. K. Ting, Proteome Sci. 9, S19 (2011). 
[26] J. McCall, J. Comput. Appl. Math. 184, 205 (2005).

[27] E. Unsal, J. H. Dane, and G. V. Dozier, Vadose Zone J. 4, 389 (2005).

[28] L. Mosser, O. Dubrule, and M. J. Blunt, Phys. Rev. E 96, 043309 (2017).

[29] M. Prat and T. Agaësse, Thin Porous Media: Handbook of Porous Media, 3rd ed., edited by K. Vafai (CRC, Boca Raton, FL, 2015), Chap. 4, pp. 89-112.
[30] R. G. Larson and N. R. Morrow, Powder Technology 30, 123 (1981).

[31] A. P. Sheppard, M. A. Knackstedt, W. V. Pinczewski, and M. Sahimi, J. Phys. A 32, L521 (1999).

[32] M. Knackstedt, M. Sahimi, and A. P. Sheppard, Phys. Rev. E 61, 4920 (2000).

[33] R. H. Brooks and A. T. Corey, J. Irrig. Drain. Div. 92, 61 (1966). 